\title{
Ethnobotanical Investigation on Medicinal Plants Traditionally Used Against Human Ailments in Ensaro District, North Shewa Zone, Amhara Regional State, Ethiopia
}

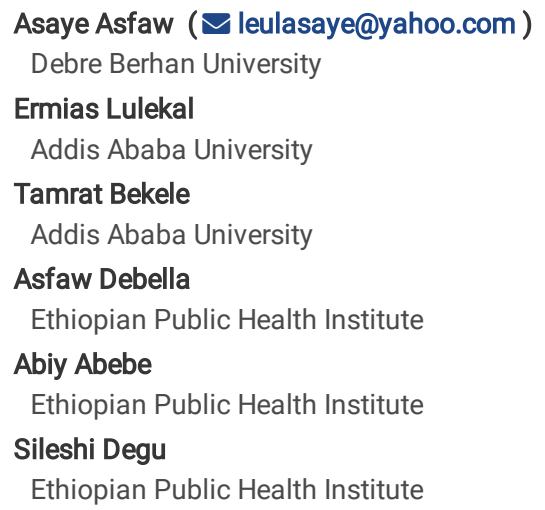




\section{Abstract}

Introduction

People all over the world have been using medicinal plants to treat various ailments and diseases throughout history. It is still common in developing countries. Because is easily accessible, affordable, and trusted by the communities. Although there are several ethnobotanical studies on medicinal plants in Ethiopia, the number of medicinal plant species and indigenous knowledge documented is still low compared to the high diversity of culture and habitats.

Methods

Ethnobotanical data were collected during the 13th of February 2019 and the 26th of October 2020 through Semi-structured interviews, field observation, a guided field walk, and focus group discussions

Result

the study documented 142 medicinal plant species distributed in 125 genera and 56 families which used to treat 52 ailments. Most of these species were collected from wild habitats. The most used plant families were Fabaceae (14) followed by lamiaceae (9) and Solanaceae (9). Most of these medicinal plants were herbs followed by shrubs.

Conclusion

The study revealed a relatively high diversity of medicinal plants that indicated the existence of indigenous knowledge of using medicinal plants to treat ailments and diseases. The findings of this study supported up the idea that indigenous knowledge of using medicinal plants is still alive in highly degraded areas. To make sustainable use of these medicinal plants in the study area, local people must collaborate with natural resource administrators to manage them before they become seriously threatened. Both ex-situ and In-situ conservation strategies should be combined and employed urgently for optimal conservation results

\section{Introduction}

For generations, the use of medicinal plants to treat and control diseases of humans and livestock is an old practice in many parts of the world. Yet, this tradition is very common in low and middle-income countries for their primary health needs (Ernst, 2000). This is because traditional medicines are used to treat diseases of humans and livestock due to economic affordability, cultural acceptability, and ease of accessibility (Teklehaymanot \& Giday, 2007; Chekole, 2017).

A wide range of traditional medicinal materials are used to treat and prevent health problems; medicinal plants, which have been used for the prevention and control of various diseases in humans and livestock since time immemorial, comprise the majority of the total (Giday et al., 2003). The use of medicinal plants is a fundamental component of traditional medicines in the African traditional health care system.

The role of medicinal plants in the traditional health care system of Ethiopia has a long history (Abebe, 1986). Yet, about $80 \%$ of the human population and $90 \%$ of livestock are dependent on traditional medicines in the country (Bekele, 2007; Assefa et al., 2010). This widespread use of traditional medicines in the country is principally due to ease of accessibility and good trust by the community (Moges \& Moges, 2019). The majority of traditional remedies in the country are medicinal plants (Abebe \& Ayehu, 1993; Abebe et al., 2001; Lulekal et al., 2008).

Ethiopia is one of the richest African countries in traditional medicines and associated indigenous knowledge which is mainly related to the presence of many ethnic groups and unique cultures in each ethnic (Mesfin et al., 2009). The presence of high diversity of plants in the country is associated with diversified climates and topography that promote diversified plant species (Kelbessa \& Demissew, 2014). In Ethiopia, there are about 800 medicinal plants (Deffar, 1998) and the majority of medicinal plants are harvested from the wild (Lulekal et al., 2013). Although the country is rich in plant biodiversity, they are threatened by a range anthropogenic activity (Mebrat \& Gashaw, 2013; Getaneh \& Girma, 2014; Lulekal et al., 2014). Therefore, the conservation and sustainable utilization of these plant species are vital.

The existence of diverse cultures with different ethnic groups in Ethiopia can contribute a large to local biodiversity conservation (Hartmann, 2008). Moreover, traditional medicinal plant species and their uses are likely to be more abundant in such cultures. However, the number of medicinal plant species and indigenous knowledge documented is still low comparing to the high diversity of culture and habitats (Getaneh \& Girma, 2014).

The community of Ensaro district lives in the high land, midland, and low land areas. As the result, the diversity of plants including medicinal plants is expected to be high. However, there has been no scientific record on such resources in Ensaro district yet. If anthropogenic activities are high on the vegetation of the study area, many medicinal plants may be threatened and lost even before documentation. According to Mebrat and Gashaw (2013), important medicinal plants are eliminating from the environment due to deforestation for fuelwood or expansion of agricultural land (Mebrat \& Gashaw, 2013). Therefore, the need to conduct ethnobotanical research to document medicinal plants and related indigenous practices is an urgent issue (Hamilton, 2003; Teklehaymanot et al., 2007). The ethnobotanical studies conducted in Ethiopia are very few as compared to the high diversity of ethnic groups with different cultures (Teklehaymanot et al., 2007). Hence, the present study is aimed to document traditional medicinal plants and associated indigenous practices used for the treatment of human diseases in Ensaro district, Amhara Regional State, Ethiopia. This is the first study of its kind in this study area, as far as our literature search. As a result, the medicinal plants used in the study districts, Ensaro district in the Amhara region, Ethiopia, were compiled in this research.

\section{Materials And Methods}




\subsection{Description of the study area and population}

Ensaro district is geographically located between $9^{\circ} 35 \rrbracket-9^{\circ} 55 \llbracket \mathrm{N}$ and $38^{\circ} 50^{\prime}-39^{\circ} 5 \llbracket E$ North Shewa Zone, Amhara Regional State, Ethiopia. The total area of the district is about $44,217.6$ ha (Figure 1). Most of the district is lowland (Figure 1) with a mean annual rainfall of $1174 \mathrm{~mm}$ and a temperature of $17.70 \mathrm{C}$. The total population is about 74312 (CSA, 2013), out of which $94.7 \%$ inhabit rural areas, deriving their livelihood from mixed agriculture (Cherinet \& Mekonnen, 2019).

Ensaro district was selected purposively for this study. This district is one of the drought-affected districts in the North Shewa Zone of Amhara Regional State. As reported by Cherinet and Mekonnen (2019), in the district there is a trend of increasing mean annual temperature and decreasing mean annual rainfall for the three decades. This harms the vegetation of the area and initiates this research to check the status of cultural knowledge regarding medicinal plants and the plant taxa in the district. There are 14867 households in the district and the major economic activity of the population is a mixed farming system. Based on the information gathered during the reconnaissance survey, the district has fourteen smaller administrative units at different distances from the administrative center (Lemi Town). All of these units were included in this ethnobotanical investigation.

\subsection{Selection of informants}

This ethnobotanical survey included 375 informants ( 271 males and 104 females) from all 13 kebeles of Ensaro district, which is the smallest administrative unit in Amhara Regional State's North Shewa Zone. Systematic random and intentional sampling techniques have been used to obtain representative informants as described by Lulekal et al. (2008) and Yineger et al. (2007). The age of informants ranged from 22-90 years. Two hundred eighty (280) general informants were systematically selected from total households and 95 key informants (77 male and 18 female) who were traditional healers purposely selected based on the recommendations of elders, local authorities, and religious leaders.

\subsection{Methods of data collection}

Ethnobotanical data were collected during the 13th of February 2019 and the 26th of October 2020 through Semi-structured interviews, field observation, a guided field walk, and focus group discussions (Martin, 1995; Cotton, 1996). The semi-structured interviews were prepared ahead of time in the English language and translated during the interview that is the mother tongue of informants. Informant interview was conducted individually (Martin, 1995; Alexiades \& Sheldon, 1996) to obtain information about medicinal plant species, parts used, preparation techniques, and common human diseases treated, administration routes and dosage. Ethnobotanical data regarding habitat, abundance, and the threat of medicinal plants were also collected. The voucher specimen of all mentioned medicinal plants during the interview were collected from different habitats with the assistance of traditional healers. Essential information such as GPS data, the local name of the plant, habitat and life forms of plant specimens were recorded. Specimens were numbered, pressed, dried, identified, and deposited at the national herbarium of Ethiopia in Addis Ababa University, Ethiopia. Plant identification was performed using the flora of Ethiopia and Eritrea. The accuracy of identification was confirmed by the comparison with the deposited authenticated specimens from Addis Ababa University Herbarium and by the help of supervisors.

\subsection{Data analysis}

Data were entered into MS Excel spreadsheet for statistical analysis. Both qualitative and quantitative using preference ranking, direct matrix ranking, and fidelity level (species consensus) were analyzed. The results were presented using tables, graphs, and percentages following Martin (1995). Fidelity level values were to determine the relative healing potential of each medicinal plant based on the proportion of informants who agreed on its use against a given disease category (Tardío \& Pardo-de-Santayana, 2008).

Fidelity level was estimated using a formula:

FL $(\%)=\frac{I_{P}}{I_{U}} \times 100$,

Where $\mathrm{FL}(\%)$ is fidelity level, IP is the number of respondents who reported the utilization of medicinal plants for a specific main ailment and IU is the total number of respondents who mentioned the same plant for any ailment (Friedman et al., 1986). This technique helps to recommend those medicinal plants having high fidelity levels are more effective that can be further analyzed for their antimicrobial activity and drug formulation (Heinrich, 2000).

Direct matrix ranking exercise was used to compare the use diversity of a given plant species using the methods proposed by Martin (1995), and Cotton (1996). The multipurpose uses of medicinal plant species were selected out of the total medicinal plants. Key informants listed the uses of these species. These key informants were asked to assign use values to each species as follows ( $5=$ best, $4=$ very good, $3=$ good, $2=$ less used, $1=$ least used and $0=$ not used). The average values (scores) given to each medicinal plant species were summed up and ranked. Moreover, the ten key informants were also involved in a priority ranking exercise that was focusing on perceived threatening factors of the medicinal plant species.

In the end, preference ranking from informants' response on ideas related to disease treatment was analyzed following (Martin, 1995). Based on their preferences ranking, they ranked individually those selected medicinal plants in treating the mentioned ailments following previous scholars (Cotton, 1996).

\section{Ethical consideration}

The study was carried out after being approved by the Ethiopian public health institute, traditional medicine directorate. Before filling the questionnaires, the participants' consent was obtained and they were assured that their responses would be used only for research purposes, and the information given would be treated with utmost care and confidentiality. 


\section{Results}

\subsection{Demographic profiles of respondents}

In this study, 375 informants (95 key informants and 280 general informants) were involved. Most of the participants were men (72.3\%) and the rest $27.7 \%$ were women. The age of participants ranges from 22 to 90 with a mean value of 48.5 years. Regarding educational status $60 \%$ of the informants were literate and the rest $40 \%$ were illiterate. $76 \%$ of the respondents were married, $11 \%$ were single, $7 \%$ were divorced and $6 \%$ were widowed.

\subsection{Diversity of medicinal plants}

The study conducted in Ensaro district recorded 142 medicinal plants (Table 2). These species are distributed across 125 genera and 56 families.

Table 1. List of medicinal plants used for human ailments: scientific name; local name (LN), family; life form (LF); diseases treated (DT); parts use(PU) conditions of use (CU); preparation methods(PM); ingredients added(IA); application methods(AP); routes of administration(RA); not recommended to(NRT) and voucher number

Twenty-nine medicinal plant families were represented by two or more species, while 27 families were represented by only a single species each. Fabaceae was the dominant family contributed by 14 species (9.9\%) followed by Lamiaceae and Solanaceae represented by nine species each. Therefore, about $81 \%$ of the families are represented by more than one species (Table 2).

Table 2.Diversity of medicinal plants in each plant family 


\begin{tabular}{|c|c|c|c|c|c|c|c|c|}
\hline Scientific name & LN & Family & LF & DT & PU & CU & PM & IA \\
\hline $\begin{array}{l}\text { Trigonella foenum- } \\
\text { graecum L. }\end{array}$ & Abish & Fabaceae & $\mathrm{H}$ & Blood pressure & Seeds & Dry & $\begin{array}{l}\text { Powdering and } \\
\text { soaking in water }\end{array}$ & Sugar \\
\hline $\begin{array}{l}\text { Dichrostachys } \\
\text { cinerea (L.) Wight \& Arn. }\end{array}$ & Ader & Fabaceae & $\mathrm{T}$ & Cellulitis & Leaves & $\mathrm{F}$ & Crushing & $\begin{array}{l}\text { Vaseli } \\
\text { butter }\end{array}$ \\
\hline $\begin{array}{l}\text { Buddleja polystachya } \\
\text { Fresen. }\end{array}$ & Afar & Buddlejaceae & S & Tonsillitis & Shoot tips & $\mathrm{F}$ & Crushing & Water \\
\hline \multirow[t]{2}{*}{ Carissa spinarum L. } & \multirow[t]{2}{*}{ Agam } & \multirow[t]{2}{*}{ Apocynaceae } & \multirow[t]{2}{*}{$\mathrm{s}$} & Snakebite & Leaves & $\mathrm{F}$ & unprocessed plant & None \\
\hline & & & & Evil eye & Root & D & $\begin{array}{l}\text { Powdering and } \\
\text { smoking }\end{array}$ & None \\
\hline $\begin{array}{l}\text { Eleusine } \\
\text { floccifolia (Forssk.) } \\
\text { Spreng. }\end{array}$ & Akrima & Poaceae & $\mathrm{H}$ & Snakebite & Root & $\mathrm{F}$ & unprocessed plant & None \\
\hline \multirow{4}{*}{$\begin{array}{l}\text { Tragia pungens (Forssk.) } \\
\text { Müll.Arg. }\end{array}$} & \multirow[t]{4}{*}{ Aleblabit } & \multirow[t]{4}{*}{ Euphorbiaceae } & \multirow[t]{4}{*}{ S } & Eczema & Leaves & $\mathrm{F}$ & Crushing & butter \\
\hline & & & & Impotence & Root & D & Powdering & $\begin{array}{l}\text { Traditi } \\
\text { bevera }\end{array}$ \\
\hline & & & & Cellulitis & Leaves & $\mathrm{F}$ & Crushing & None \\
\hline & & & & Scorpion bite & Root & $\mathrm{F}$ & Crushing & none \\
\hline $\begin{array}{l}\text { Abutilon longicuspe } \\
\text { A.Rich. }\end{array}$ & Amedmado & Malvaceae & s & Wound & Leaves & $\mathrm{F}$ & Crushing & None \\
\hline Cyathula cylindrica Moq. & Arefrafo & Amaranthaceae & $\mathrm{H}$ & Bleeding & Leaves & $\mathrm{F}$ & Crushing & None \\
\hline \multirow{2}{*}{$\begin{array}{l}\text { CyphoStemma } \\
\text { adenocaulis (Steud. ex } \\
\text { A.Rich.) Desc. ex Wild \& } \\
\text { R.B.Drumm. }\end{array}$} & \multirow[t]{2}{*}{$\begin{array}{l}\text { Aserkush } \\
\text { tebetebkush }\end{array}$} & \multirow[t]{2}{*}{ Vitaceae } & \multirow[t]{2}{*}{$\mathrm{H}$} & Rabies & Root & D & Powdering & Coffe $\epsilon$ \\
\hline & & & & Wound & Root & D & Powdering & Butter \\
\hline $\begin{array}{l}\text { Galium } \\
\text { aparinoides Forssk. }\end{array}$ & Ashkit & Rubiaceae & $\mathrm{H}$ & Wound & Root & $\mathrm{F}$ & Crushing & None \\
\hline Datura stramonium L. & Astenagrit & Solanaceae & $\mathrm{H}$ & Dandruff & Leaves & $\mathrm{F}$ & Crushing & None \\
\hline Verbena officinalis $L$. & Atuch & Verbenaceae & $\mathrm{H}$ & Diarrhoea & Root & $\mathrm{F}$ & Crushing & water \\
\hline \multirow{4}{*}{$\begin{array}{l}\text { Clematis } \\
\text { simensis Fresen. }\end{array}$} & \multirow[t]{4}{*}{ Azo hareg } & \multirow[t]{4}{*}{ Ranunculaceae } & \multirow[t]{4}{*}{$C$} & Hemorroids & Leaves & D & Powdering & Vaseli \\
\hline & & & & Cellulitis & Leaves & D & Crushing & Vaseli \\
\hline & & & & Wound & Leaves & $\mathrm{F}$ & Powdering & Salt \\
\hline & & & & Eczema & Leaves & D & Crushing & Butter \\
\hline Ficus palmata Forssk. & Beles & Euphorbiaceae & S & Wound & Stem bark & $\mathrm{F}$ & Crushing & None \\
\hline \multirow[t]{2}{*}{ Capsicum annuum L. } & \multirow[t]{2}{*}{ Berberie } & \multirow[t]{2}{*}{ Solanaceae } & \multirow[t]{2}{*}{$\mathrm{H}$} & Scorpion bite & Leaves & $\mathrm{F}$ & Crushing & None \\
\hline & & & & Typhoid & Fruits & F/D & Powdering/Crushing & Water \\
\hline \multirow{2}{*}{$\begin{array}{l}\text { Millettia } \\
\text { ferruginea (Hochst.) Bak. }\end{array}$} & \multirow[t]{2}{*}{ Birbira } & \multirow[t]{2}{*}{ Fabaceae } & \multirow[t]{2}{*}{$\mathrm{T}$} & Eczema & Seeds & D & Powdering & Butter \\
\hline & & & & Wound & Leaves & D & Powdering & Vaseli \\
\hline \multirow{3}{*}{$\begin{array}{l}\text { Croton } \\
\text { macrostachyus Hochst. } \\
\text { ex Del. }\end{array}$} & \multirow[t]{3}{*}{ Bisana } & \multirow[t]{3}{*}{ Euphorbiaceae } & $\mathrm{T}$ & Cellulitis & Leaves & $\mathrm{F}$ & Crushing & Vaseli \\
\hline & & & & Evil eye & Root & D & Powdering & None \\
\hline & & & & Mitch & Leaves & $\mathrm{F}$ & Boiling in water & None \\
\hline Catha edulis (Vahl) Endl. & Chat & Celastraceae & S & Snakebite & Leaves & $\mathrm{F}$ & unprocessed plant & none \\
\hline Premna schimperi Engl. & Checho & Lamiaceae & s & Cellulitis & Leaves & D & Powdering & Vaseli \\
\hline $\begin{array}{l}\text { Sida schimperiana } \\
\text { Hochst. ex A.Rich. }\end{array}$ & Chifrig & Malvaceae & $\mathrm{H}$ & "Likft" (devil illness) & Root & D & $\begin{array}{l}\text { Powdering and } \\
\text { smoking }\end{array}$ & None \\
\hline & & & & Cellulitis & Root & $\mathrm{F}$ & Crushing & None \\
\hline $\begin{array}{l}\text { Artemisia } \\
\text { abyssinica Sch. Bip. ex } \\
\text { A. Rich. }\end{array}$ & Chiqugn & Asteraceae & $\mathrm{H}$ & Evil eye & Leaves & D & $\begin{array}{l}\text { Powdering and } \\
\text { smoking }\end{array}$ & None \\
\hline
\end{tabular}




\begin{tabular}{|c|c|c|c|c|c|c|c|c|}
\hline Scientific name & LN & Family & LF & DT & PU & $\mathrm{CU}$ & PM & IA \\
\hline $\begin{array}{l}\text { Plectranthus } \\
\text { cylindraceus Hochst. ex } \\
\text { Benth. }\end{array}$ & Dachet & Lamiaceae & $\mathrm{H}$ & wound & Leaves & $\mathrm{F}$ & Heating & None \\
\hline $\begin{array}{l}\text { Ocimum } \\
\text { lamiifolium Hochst. ex } \\
\text { Benth. }\end{array}$ & Damakessie & Lamiaceae & $S$ & Mitch & Leaves & $\mathrm{F}$ & Boiling in water & None \\
\hline Euclea divinorum Hiern & Dedeho & Ebenaceae & S & Wound & Root & $\mathrm{D}$ & Powdering & Vaseli \\
\hline Solanum villosum Mill. & Derekus & Solanaceae & $S$ & Eye disease & Leaves & $\mathrm{F}$ & $\begin{array}{l}\text { Crushing and } \\
\text { squeezing }\end{array}$ & None \\
\hline $\begin{array}{l}\text { Acacia etbaica } \\
\text { Schweinf. }\end{array}$ & Derie & Fabaceae & $\mathrm{T}$ & Scabies & Leaves & $\mathrm{F}$ & Crushing & Water \\
\hline \multirow[t]{3}{*}{$\begin{array}{l}\text { Calpurnia aurea (Ait.) } \\
\text { Benth. }\end{array}$} & \multirow[t]{3}{*}{ Digita } & \multirow[t]{3}{*}{ Fabaceae } & \multirow[t]{3}{*}{$\mathrm{S}$} & Jaundice & Seeds & $\mathrm{D}$ & $\begin{array}{l}\text { Powdering and } \\
\text { baking } \\
\text { with Eragrostis tef }\end{array}$ & None \\
\hline & & & & Diarrhea & leaves & $\mathrm{F}$ & $\begin{array}{l}\text { Crushing and } \\
\text { boiling }\end{array}$ & Water \\
\hline & & & & Rabies & Seeds & $\mathrm{D}$ & $\begin{array}{l}\text { Powdering and } \\
\text { baking }\end{array}$ & Eragro \\
\hline Coriandrum sativum L. & Dinbilal & Apiaceae & $\mathrm{H}$ & Blood pressure & Seeds & $\mathrm{D}$ & $\begin{array}{l}\text { Powdering and } \\
\text { boiling }\end{array}$ & Coffee \\
\hline $\begin{array}{l}\text { Taverniera } \\
\text { abyssinica A. Rich. }\end{array}$ & Dingetegna & Fabaceae & $\mathrm{H}$ & $\begin{array}{l}\text { "Megagna” ( unidentified } \\
\text { gastrointestinal } \\
\text { disorder) }\end{array}$ & Root & $\mathrm{D}$ & $\begin{array}{l}\text { Powdering and } \\
\text { smoking }\end{array}$ & None \\
\hline Ferula communis L. & Dog & Apiaceae & $\mathrm{H}$ & Diarrhoea & Root & $\mathrm{F}$ & Crushing & Water \\
\hline $\begin{array}{l}\text { Syzygium } \\
\text { guineense (Wild.) DC. }\end{array}$ & Doqima & Myrtaceae & $\mathrm{T}$ & Evil eye & Root & D & $\begin{array}{l}\text { Powdering and } \\
\text { smoking }\end{array}$ & None \\
\hline \multirow[t]{4}{*}{ Rumex nervosus Vahl } & \multirow[t]{4}{*}{ Embuacho } & \multirow[t]{4}{*}{ Polygonaceae } & \multirow[t]{4}{*}{$S$} & Circumcise infection & Leaves & $\mathrm{F}$ & Crushing & None \\
\hline & & & & Delayed placenta & Leaves & $\mathrm{F}$ & Crushing & Water \\
\hline & & & & Evil eye & Shoot tips & $\mathrm{F}$ & unprocessed plant & None \\
\hline & & & & Cellulitis & Leaves & $\mathrm{F}$ & Crushing & None \\
\hline $\begin{array}{l}\text { Kalanchoe marmorata } \\
\text { Baker }\end{array}$ & Enchibchubit & Crassulaceae & $\mathrm{H}$ & Tumor & Leaves & $\mathrm{F}$ & Heating & None \\
\hline $\begin{array}{l}\text { Kalanchoe petitiana A. } \\
\text { Rich. }\end{array}$ & Endahahula & Crassulaceae & $\mathrm{H}$ & Tumor & Leaves & $\mathrm{F}$ & Heating & None \\
\hline \multirow{5}{*}{$\begin{array}{l}\text { Phytolacca } \\
\text { dodecandra L' Her. }\end{array}$} & \multirow[t]{5}{*}{ Mekan endod } & \multirow[t]{5}{*}{ Phytolaccaceae } & \multirow[t]{5}{*}{$\mathrm{S}$} & Rabies & Root & $\mathrm{F}$ & Crushing & Milk \\
\hline & & & & Abortion & Leaves & $\mathrm{F}$ & Crushing & Water \\
\hline & & & & Leprosy & Leaves & $\mathrm{F}$ & $\begin{array}{l}\text { Crushing and } \\
\text { soaking in water }\end{array}$ & Salt \\
\hline & & & & Jaundice & Root & $\mathrm{D}$ & Powdering & “Injera \\
\hline & & & & Syphilis & Leaves & $\mathrm{F}$ & Crushing & water \\
\hline Ximenia americana L. & Enkoy & Olacaceae & $\mathrm{T}$ & Jaundice & $\begin{array}{l}\text { Leaves/stem } \\
\text { bark }\end{array}$ & $\begin{array}{l}\mathrm{F} \\
/ \mathrm{D}\end{array}$ & $\begin{array}{l}\text { Crushing } \\
\text { /powdering }\end{array}$ & Water \\
\hline Foeniculum vulgare Mill & Ensilal & Apiaceae & $\mathrm{H}$ & $\begin{array}{l}\text { "Mognbagegn" } \\
\text { (Anthrax) }\end{array}$ & Leaves & $\mathrm{F}$ & Crushing & Water \\
\hline $\begin{array}{l}\text { Leonotis } \\
\text { ocymifolia (Burm.) } \\
\text { Iwarsson var. raineriana }\end{array}$ & Eras kebdo & Lamiaceae & $S$ & Cellulitis & Leaves & D & Powdering & Vaseli \\
\hline \multirow[t]{3}{*}{$\begin{array}{l}\text { Securidaca } \\
\text { longipedunculata Fresen }\end{array}$} & \multirow[t]{3}{*}{ Este-menahie } & \multirow[t]{3}{*}{ Polygalaceae } & \multirow[t]{3}{*}{$S$} & $\begin{array}{l}\text { “Megagna” ( } \\
\text { unidentified }\end{array}$ & Root & $\mathrm{D}$ & $\begin{array}{l}\text { Powdering and } \\
\text { smoking }\end{array}$ & None \\
\hline & & & & $\begin{array}{l}\text { gastrointestinal } \\
\text { disorder) }\end{array}$ & & & & \\
\hline & & & & Mitch & Root & $\mathrm{D}$ & $\begin{array}{l}\text { Powdering and } \\
\text { smoking }\end{array}$ & None \\
\hline
\end{tabular}

Page 6/27 


\begin{tabular}{|c|c|c|c|c|c|c|c|c|}
\hline Scientific name & $\mathrm{LN}$ & Family & LF & DT & PU & $\mathrm{CU}$ & PM & IA \\
\hline \multirow{4}{*}{$\begin{array}{l}\text { Andrachne aspera } \\
\text { Spreng. }\end{array}$} & \multirow[t]{4}{*}{ Este-tekezie } & \multirow[t]{4}{*}{ Euphorbiaceae } & \multirow[t]{4}{*}{$\mathrm{H}$} & Eczema & Root & $F / D$ & Powdering & Vaseli \\
\hline & & & & Tonsillitis & Root & $F / D$ & unprocessed plant & None \\
\hline & & & & Evil eye & Root & $\mathrm{D}$ & $\begin{array}{l}\text { Powdering and } \\
\text { smoking }\end{array}$ & None \\
\hline & & & & Snakebite & Root & $F / D$ & unprocessed plant & None \\
\hline $\begin{array}{l}\text { Emilia sonchifolia (L.) } \\
\text { DC. ex DC. }\end{array}$ & $\begin{array}{l}\text { Etse hayu este } \\
\text { yikestil }\end{array}$ & Asteraceae & $\mathrm{H}$ & Impotence & Root & $\mathrm{F}$ & unprocessed plant & None \\
\hline $\begin{array}{l}\text { Polygala } \\
\text { abyssinica R.Br. ex } \\
\text { Fresen. }\end{array}$ & Etse libona & Polygalaceae & $\mathrm{H}$ & Snakebite & Leaves & $\mathrm{F}$ & unprocessed plant & None \\
\hline Lepidium sativum L. & Feto & Brassicaceae & $\mathrm{H}$ & Mitch & Seeds & $\mathrm{D}$ & $\begin{array}{l}\text { Powdering and } \\
\text { boiling }\end{array}$ & Coffee \\
\hline \multirow{2}{*}{$\begin{array}{l}\text { Clutia abyssinica Jaub. } \\
\text { and Spach. }\end{array}$} & \multirow[t]{2}{*}{ Fyelefech } & \multirow[t]{2}{*}{ Peraceae } & \multirow[t]{2}{*}{ S } & Diarrhoea & Leaves & $\mathrm{F}$ & Crushing & Coffe $\epsilon$ \\
\hline & & & & Dandruff & Leaves & $F / D$ & Crushing/powdering & Butter, \\
\hline \multirow{4}{*}{$\begin{array}{l}\text { Ziziphus spina- } \\
\text { christi (L.) Desf. }\end{array}$} & \multirow[t]{4}{*}{ Geba } & \multirow[t]{4}{*}{ Rhamnaceae } & \multirow[t]{4}{*}{$\mathrm{T}$} & Abdominal pain & Stem bark & $\mathrm{F}$ & unprocessed plant & None \\
\hline & & & & scorpion bite & Leaves & $\mathrm{F}$ & Crushing & None \\
\hline & & & & Snakebite & $\begin{array}{l}\text { Stem } \\
\text { bark/Root } \\
\text { bark }\end{array}$ & $\mathrm{F}$ & unprocessed plant & None \\
\hline & & & & Diarrhoea & Stem bark & $\mathrm{F}$ & unprocessed plant & None \\
\hline $\begin{array}{l}\text { Solanum marginatum L. } \\
\text { f. }\end{array}$ & Geber embuay & Solanaceae & $S$ & Scabies & Fruit & $\mathrm{F}$ & unprocessed plan & None \\
\hline $\begin{array}{l}\text { Otostegia fruticosa } \\
\text { (Forssk.) Schweinf. ex } \\
\text { Penzig }\end{array}$ & Geram tunjit & Lamiaceae & $S$ & $\begin{array}{l}\text { Abdominal pain after } \\
\text { birth }\end{array}$ & Leaves & $\mathrm{F}$ & Crushing & Water \\
\hline \multirow{3}{*}{$\begin{array}{l}\text { Kedrostis } \\
\text { foetidissima (Jacq.) } \\
\text { Cogn }\end{array}$} & \multirow{3}{*}{$\begin{array}{l}\text { Gim } \\
\text { hareg/yewusha } \\
\text { fes/ }\end{array}$} & \multirow[t]{3}{*}{ Cucurbitaceae } & \multirow[t]{3}{*}{ C } & Wound & Leaves & $\mathrm{F}$ & Crushing & Lemor \\
\hline & & & & Evil eye & Root & $\mathrm{D}$ & $\begin{array}{l}\text { Powdering and } \\
\text { smoking }\end{array}$ & None \\
\hline & & & & Delayed placenta & Leaves & $\mathrm{F}$ & Crushing & Water \\
\hline $\begin{array}{l}\text { Psydrax } \\
\text { schimperiana (A.Rich.) } \\
\text { Bridson }\end{array}$ & Gimie seged & Rubiaceae & $S$ & Snakebite & Leaves & $\mathrm{F}$ & Crushing & Water \\
\hline $\begin{array}{l}\text { CyphoStemma } \\
\text { cyphopetalum (Fresen.) } \\
\text { Desc. ex Wild \& } \\
\text { R.B.Drumm. }\end{array}$ & Gindosh & Vitaceae & $\mathrm{H}$ & Snakebite & $\begin{array}{l}\text { Stem/root } \\
\text { barks }\end{array}$ & $\mathrm{F}$ & unprocessed plant & None \\
\hline \multirow[t]{3}{*}{$\begin{array}{l}\text { Withania somnifera (L.) } \\
\text { Dunal }\end{array}$} & \multirow[t]{3}{*}{ Gizewa } & \multirow[t]{3}{*}{ Solanaceae } & \multirow[t]{3}{*}{$\mathrm{H}$} & Evil eye & Root & D & $\begin{array}{l}\text { Powdering and } \\
\text { smoking }\end{array}$ & None \\
\hline & & & & $\begin{array}{l}\text { "Megagna"( unidentified } \\
\text { gastrointestinal } \\
\text { disorder) }\end{array}$ & Root & D & $\begin{array}{l}\text { Powdering and } \\
\text { smoking }\end{array}$ & None \\
\hline & & & & Mitch & Root & $\mathrm{D}$ & $\begin{array}{l}\text { Powdering and } \\
\text { smoking }\end{array}$ & None \\
\hline \multirow[t]{4}{*}{$\begin{array}{l}\text { Inula confertiflora A. } \\
\text { Rich. }\end{array}$} & \multirow[t]{4}{*}{ Gobez teqes } & \multirow[t]{4}{*}{ Asteraceae } & \multirow[t]{4}{*}{$\mathrm{S}$} & Eye disease & Leaves & $\mathrm{F}$ & $\begin{array}{l}\text { Crushing and } \\
\text { squeezing }\end{array}$ & None \\
\hline & & & & Rabies & Leaves & $\mathrm{F}$ & Crushing & Water \\
\hline & & & & Evil eye & Root & D & $\begin{array}{l}\text { Powdering and } \\
\text { smoking }\end{array}$ & None \\
\hline & & & & Jaundice & Leaves & $\mathrm{F}$ & Crushing & Water \\
\hline $\begin{array}{l}\text { Vernonia } \\
\text { amygdalina Del. }\end{array}$ & Grawa & Asteraceae & $S$ & $\begin{array}{l}\text { "Megagna" (unidentified } \\
\text { gastrointestinal } \\
\text { disorder) }\end{array}$ & Leaves & $\mathrm{F}$ & Crushing & Water \\
\hline $\begin{array}{l}\text { Senna singueana (Del.) } \\
\text { Lock }\end{array}$ & Gufa & Fabaceae & $S$ & Jaundice & Seeds & $\mathrm{D}$ & Powdering & Water \\
\hline
\end{tabular}

Page $7 / 27$ 


\begin{tabular}{|c|c|c|c|c|c|c|c|c|}
\hline Scientific name & LN & Family & LF & DT & PU & $\mathrm{CU}$ & PM & IA \\
\hline & & & & Abdominal pain & Stem bark & $\mathrm{F}$ & unprocessed plant & None \\
\hline & & & & Snakebite & Stem bark & $\mathrm{F}$ & unprocessed plant & None \\
\hline & & & & Cellulitis & Leaves & $\mathrm{F}$ & Crushing & Water \\
\hline \multirow[t]{3}{*}{ Ricinus communis L. } & \multirow[t]{3}{*}{ Gulo } & \multirow[t]{3}{*}{ Euphorbiaceae } & \multirow[t]{3}{*}{$\mathrm{H}$} & Eye disease & Root & $\mathrm{F}$ & Crushing & Water \\
\hline & & & & Wound & Root & $\mathrm{D}$ & Powdering & Vaseli \\
\hline & & & & Infant dysentery & Leaves & $\mathrm{F}$ & Crushing & Water \\
\hline \multirow[t]{2}{*}{$\begin{array}{l}\text { Acacia oerfota } \\
\text { (Forssk.) Schweinf. }\end{array}$} & \multirow[t]{2}{*}{ Gumero } & \multirow[t]{2}{*}{ Fabaceae } & \multirow[t]{2}{*}{$S$} & Evil eye & Root & $\mathrm{D}$ & $\begin{array}{l}\text { Powdering and } \\
\text { smoking }\end{array}$ & None \\
\hline & & & & $\begin{array}{l}\text { Excessive bleeding of } \\
\text { menstruation }\end{array}$ & Leaves & $F / D$ & Powdering & Water \\
\hline \multirow[t]{5}{*}{ Zehneria scabra Sond. } & \multirow[t]{5}{*}{ Hareg eresa } & \multirow[t]{5}{*}{ Cucurbitaceae } & \multirow[t]{5}{*}{ C } & Cellulitis & Leaves & $\mathrm{F}$ & Crushing & Vaseli \\
\hline & & & & Scabies & Leaves & $\mathrm{D}$ & Powdering & Vaseli \\
\hline & & & & $\begin{array}{l}\text { Excessive bleeding of } \\
\text { menstruation }\end{array}$ & Leaves & $\mathrm{F}$ & Crushing & Water \\
\hline & & & & Likft (devil illness) & whole part & $\mathrm{D}$ & $\begin{array}{l}\text { Powdering and } \\
\text { smoking }\end{array}$ & None \\
\hline & & & & Mitch & Leaves & $\mathrm{F}$ & Crushing & None \\
\hline \multirow[t]{8}{*}{ Salvia nilotica Jacq. } & \multirow[t]{8}{*}{ Hulgeb } & \multirow[t]{8}{*}{ Lamiaceae } & \multirow[t]{8}{*}{$\mathrm{H}$} & Mitch & whole part & $\mathrm{F}$ & Crushing & None \\
\hline & & & & Tonsillitis & Root & $\mathrm{F}$ & unprocessed plant & None \\
\hline & & & & $\begin{array}{l}\text { When insects entered } \\
\text { ears }\end{array}$ & Leaves & $\mathrm{F}$ & $\begin{array}{l}\text { Crushing and } \\
\text { squeezing }\end{array}$ & None \\
\hline & & & & Abdominal pain & whole part & $\mathrm{F}$ & Crushing & Water \\
\hline & & & & Cellulitis & Leaves & $\mathrm{F}$ & Crushing & None \\
\hline & & & & $\begin{array}{l}\text { Excessive bleeding of } \\
\text { menstruation }\end{array}$ & Root & $\mathrm{F}$ & Crushing & None \\
\hline & & & & Wound & Leaves & $\mathrm{F}$ & Crushing & None \\
\hline & & & & Diarrhoea & Root & $\mathrm{F}$ & Crushing & Lemor \\
\hline Solanum dulcamara L. & $\begin{array}{l}\text { Karya mesel } \\
\text { yekusil } \\
\text { medhanit }\end{array}$ & Solanaceae & $\mathrm{H}$ & Wound & Leaves & $\mathrm{F}$ & Crushing & None \\
\hline Myrsine africana L. & Kechemo & Myrsinaceae & $S$ & Taeniasis & Leaves & $\mathrm{F}$ & unprocessed plant & None \\
\hline \multirow[t]{3}{*}{ Euphorbia tirucalli L. } & \multirow[t]{3}{*}{ Kinchib } & \multirow[t]{3}{*}{ Euphorbiaceae } & \multirow[t]{3}{*}{ S } & Wound & Epiphyte & $\mathrm{F}$ & Crushing & None \\
\hline & & & & Evil eye & Root & $\mathrm{D}$ & $\begin{array}{l}\text { Powdering and } \\
\text { smoking }\end{array}$ & None \\
\hline & & & & Hemorroids & Latex & $\mathrm{F}$ & Squeezing & None \\
\hline \multirow{2}{*}{$\begin{array}{l}\text { Dodonaea } \\
\text { angustifolia L. f. }\end{array}$} & \multirow[t]{2}{*}{ Kitkita } & \multirow[t]{2}{*}{ Sapindaceae } & \multirow[t]{2}{*}{$\mathrm{S}$} & Eczema & Leaves & $\mathrm{D}$ & Powdering & Vaseli \\
\hline & & & & Fire burn & Leaves & $\mathrm{D}$ & Powdering & Vaseli \\
\hline $\begin{array}{l}\text { Pterolobium } \\
\text { stellatum (Forssk.) } \\
\text { Brenan }\end{array}$ & Kontir & Fabaceae & $S$ & Evil eye & Root & $\mathrm{D}$ & $\begin{array}{l}\text { Powdering and } \\
\text { smoking }\end{array}$ & $\begin{array}{l}\text { Other I } \\
\text { plants }\end{array}$ \\
\hline $\begin{array}{l}\text { Urera } \\
\text { hypselodendron (Hochst. } \\
\text { ex A. Rich.) Wedd. }\end{array}$ & Lanquso & Utricaceae & $S$ & Wound & Stem bark & $\mathrm{F}$ & Crushing & None \\
\hline \multirow{2}{*}{$\begin{array}{l}\text { Grewia } \\
\text { ferruginea Hochst. ex } \\
\text { A.Rich. }\end{array}$} & \multirow[t]{2}{*}{ lenquata } & \multirow[t]{2}{*}{ Malvaceae } & S & Dandruff & Stem bark & $\mathrm{F}$ & Crushing & None \\
\hline & & & & Wound & Stem bark & $\mathrm{F}$ & Crushing & None \\
\hline $\begin{array}{l}\text { Adenia } \\
\text { cissampeloides (Planch. } \\
\text { ex Hook.) Harms }\end{array}$ & Leslasa hareg & Passifloraceae & C & Cellulitis & Leaves & $\mathrm{F}$ & Crushing & None \\
\hline Malva parviflora L. & Lut nechu & Malvaceae & $\mathrm{H}$ & Abdominal pain & Root & $\mathrm{F}$ & Crushing & Water \\
\hline $\begin{array}{l}\text { Rumex } \\
\text { nepalensis Spreng. }\end{array}$ & Lut qeyu & Polygonaceae & $\mathrm{H}$ & Snakebite & Root & $\mathrm{F}$ & unprocessed plant & None \\
\hline
\end{tabular}




\begin{tabular}{|c|c|c|c|c|c|c|c|c|}
\hline Scientific name & LN & Family & LF & DT & PU & $\mathrm{CU}$ & PM & IA \\
\hline & & & & "Megagna” (unidentified & Root & $\mathrm{F}$ & unprocessed plant & None \\
\hline & & & & $\begin{array}{l}\text { gastrointestinal } \\
\text { disorder) }\end{array}$ & & & & \\
\hline & & & & Abdominal pain & Root & $\mathrm{F}$ & $\begin{array}{l}\text { Crushing and } \\
\text { squeezing }\end{array}$ & Water \\
\hline \multirow[t]{2}{*}{$\begin{array}{l}\text { Rumex abyssinicus } \\
\text { Jacq. }\end{array}$} & \multirow[t]{2}{*}{ Meqmeqo } & \multirow[t]{2}{*}{ Polygonaceae } & \multirow[t]{2}{*}{$\mathrm{H}$} & Asthma & Rhizome & $\mathrm{D}$ & $\begin{array}{l}\text { Powdering and } \\
\text { boiling }\end{array}$ & Honey \\
\hline & & & & Blood pressure & Rhizome & $\mathrm{D}$ & $\begin{array}{l}\text { Powdering and } \\
\text { boiling }\end{array}$ & Tea \\
\hline Embelia schimperi Vatke & Meterie & Myrsinaceae & $S$ & Taeniasis & Fruit & $\mathrm{D}$ & Powdering & $\begin{array}{l}\text { Guizot } \\
\text { abyssi } \\
\text { "injera }\end{array}$ \\
\hline $\begin{array}{l}\text { Arisaema } \\
\text { schimperianum schott }\end{array}$ & Milas golgul & Araceae & C & Snakebite & Root & $\mathrm{F}$ & unprocessed plant & None \\
\hline \multirow{3}{*}{$\begin{array}{l}\text { Acokanthera } \\
\text { schimperi (A. DC.) Benth. } \\
\text { \& Hook.f. ex Schweinf. }\end{array}$} & \multirow[t]{3}{*}{ Mirez } & \multirow[t]{3}{*}{ Apocynaceae } & \multirow[t]{3}{*}{ S } & Evil eye & Root & $\mathrm{D}$ & Powdering & None \\
\hline & & & & Epilepsy & Root & $F / D$ & unprocessed plant & None \\
\hline & & & & Jaundice & Leaves & $\mathrm{F}$ & Crushing & Water \\
\hline \multirow{3}{*}{$\begin{array}{l}\text { Clerodendrum } \\
\text { myricoides (Hochst.) } \\
\text { R.Br. ex Vatke }\end{array}$} & \multirow[t]{3}{*}{ Misirich } & \multirow[t]{3}{*}{ Verbenaceae } & \multirow[t]{3}{*}{$S$} & Jaundice & Leaves & $\mathrm{F}$ & $\begin{array}{l}\text { Crushing and } \\
\text { squeezing }\end{array}$ & Water \\
\hline & & & & Circumcise infection & Leaves & $\mathrm{F}$ & Crushing & None \\
\hline & & & & Cellulitis & Leaves & $F / D$ & Powdering & Vaseli \\
\hline Duranta erecta L. & Muatosh & Verbenaceae & $S$ & Wound & Leaves & $\mathrm{F}$ & Crushing & Salt \\
\hline \multirow{2}{*}{$\begin{array}{l}\text { Snowdenia } \\
\text { polystachya (Fresen.) } \\
\text { Pilg. }\end{array}$} & \multirow[t]{2}{*}{ Muja } & \multirow[t]{2}{*}{ Poaceae } & \multirow[t]{2}{*}{$\mathrm{H}$} & Retained placenta & Root & $\mathrm{F}$ & Crushing & Water \\
\hline & & & & Wound & Aerial part & $\mathrm{F}$ & Crushing & None \\
\hline $\begin{array}{l}\text { Albizia } \\
\text { anthelmintica Brongn. }\end{array}$ & Musina & Fabaceae & $S$ & Taeniasis & Stem bark & D & Powdering & $\begin{array}{l}\text { Sesan } \\
\text { "injera }\end{array}$ \\
\hline \multirow{2}{*}{$\begin{array}{l}\text { Maytenus } \\
\text { senegalensis (Lam.) } \\
\text { Exell }\end{array}$} & \multirow[t]{2}{*}{ Nech atat } & \multirow[t]{2}{*}{ Celastraceae } & \multirow[t]{2}{*}{$S$} & dog bite & Leaves & $\mathrm{F}$ & Crushing & None \\
\hline & & & & Cellulitis & Leaves & $\mathrm{F}$ & Crushing & Water \\
\hline \multirow{2}{*}{$\begin{array}{l}\text { Eucalyptus } \\
\text { globulus Labill. }\end{array}$} & \multirow[t]{2}{*}{ Nech bahir zaf } & \multirow[t]{2}{*}{ Myrtaceae } & \multirow[t]{2}{*}{$\mathrm{T}$} & Mitch & Leaves & $\mathrm{F}$ & Boiling & None \\
\hline & & & & Cough & Leaves & $\mathrm{F}$ & Boiling & None \\
\hline \multirow[t]{7}{*}{ Allium sativum L. } & \multirow[t]{7}{*}{ Nech shinkurt } & \multirow[t]{7}{*}{ Amaryllidaceae } & $\mathrm{H}$ & Malaria & Bulb & $F / D$ & unprocessed plant & None \\
\hline & & & & Scabies & Bulb & $\mathrm{F} / \mathrm{D}$ & Crushing & Salt ar \\
\hline & & & & $\begin{array}{l}\text { "Mognbagegn" (disease } \\
\text { anthrax) }\end{array}$ & Bulb & $F / D$ & unprocessed plant & None \\
\hline & & & & Blood pressure & Bulb & $F / D$ & unprocessed plant & None \\
\hline & & & & Typhoid & Bulb & $F / D$ & Cutting in to pieces & Soakir \\
\hline & & & & Abdominal pain & Bulb & F/D & Unprocessed plant & None \\
\hline & & & & Hemorroids & Bulb & $F / D$ & unprocessed plant & None \\
\hline $\begin{array}{l}\text { Echinops } \\
\text { kebericho Mesfin }\end{array}$ & Qebericho & Asteraceae & $\mathrm{H}$ & Evil eye & Root & $\mathrm{D}$ & $\begin{array}{l}\text { Powdering and } \\
\text { smoking }\end{array}$ & None \\
\hline $\begin{array}{l}\text { Calotropis procera (Ait) } \\
\text { Ait. f. }\end{array}$ & Qebo & Asclepiadaceae & S & Wart & Latex & $\mathrm{F}$ & unprocessed latex & None \\
\hline $\begin{array}{l}\text { Kanahia laniflora } \\
\text { (Forssk.) R.Br. }\end{array}$ & Qechinie & Apocynaceae & S & Snakebite & Root & $\mathrm{F}$ & unprocessed root & None \\
\hline Allium cepa L. & Qey shinkurt & Amaryllidaceae & $\mathrm{H}$ & Blood pressure & Bulb & $F / D$ & Crushing & Water \\
\hline Lagenaria & Qil & Cucurbitaceae & C & Dandruff & Leaves & $\mathrm{F}$ & Crushing & Water \\
\hline Standl. & & & & Jaundice & Leaves & $\mathrm{F}$ & Crushing & None \\
\hline & & & & Dysentery in infants & Leaves & $\mathrm{F}$ & Crushing & None \\
\hline
\end{tabular}




\begin{tabular}{|c|c|c|c|c|c|c|c|c|}
\hline Scientific name & $\mathrm{LN}$ & Family & LF & DT & PU & $\mathrm{CU}$ & PM & IA \\
\hline \multirow[t]{3}{*}{$\begin{array}{l}\text { Euphorbia } \\
\text { abyssinica J.F.Gmel. }\end{array}$} & \multirow[t]{3}{*}{$\begin{array}{l}\text { Qulqual bale } \\
\text { demu }\end{array}$} & \multirow[t]{3}{*}{ Euphorbiaceae } & \multirow[t]{3}{*}{$S$} & Herpes zoster & Stem juice & $\mathrm{D}$ & $\begin{array}{l}\text { Heating and } \\
\text { Powdering }\end{array}$ & None \\
\hline & & & & Malaria & Stem juice & $\mathrm{F}$ & Baking & $\begin{array}{l}\text { traditic } \\
\text { (injera }\end{array}$ \\
\hline & & & & Leishmaniasis & Stem juice & $\mathrm{F}$ & unprocessed plant & None \\
\hline \multirow[t]{2}{*}{ Schinus molle L. } & \multirow[t]{2}{*}{ qundo berberie } & \multirow[t]{2}{*}{ Anacardiaceae } & \multirow[t]{2}{*}{$\mathrm{T}$} & Ascariasis & Root & $\mathrm{F}$ & Crushing & None \\
\hline & & & & “Mognbagegn”(Anthrax) & Seeds & $\mathrm{D}$ & $\begin{array}{l}\text { Powdering and } \\
\text { boiling in water }\end{array}$ & None \\
\hline $\begin{array}{l}\text { Urtica simensis Hochst. } \\
\text { ex A.Rich. }\end{array}$ & Sama & Utricaceae & $\mathrm{H}$ & Stomach-ache & Leaves & $\mathrm{F}$ & Roasting & Water \\
\hline \multirow{3}{*}{$\begin{array}{l}\text { Justicia } \\
\text { schimperiana (Hochst. } \\
\text { ex Nees) T. Anderson }\end{array}$} & \multirow[t]{3}{*}{ Sensel } & \multirow[t]{3}{*}{ Acanthaceae } & \multirow[t]{3}{*}{$S$} & Hemorroids & Leaves & $\mathrm{F}$ & Crushing & Water \\
\hline & & & & Likft (devil illness) & Root & D & $\begin{array}{l}\text { Powdering and } \\
\text { smoking }\end{array}$ & None \\
\hline & & & & Jaundice & Leaves & $\mathrm{F}$ & Crushing & Lemor \\
\hline $\begin{array}{l}\text { Cynodon dactylon (L.) } \\
\text { Pers. var. dactylon }\end{array}$ & Serdo & Poaceae & $\mathrm{H}$ & Rh-factor & Root & $\mathrm{D}$ & Powdering & Bread \\
\hline \multirow{5}{*}{$\begin{array}{l}\text { Aloe pulcherrima Gilbert } \\
\text { \& Sebsebe }\end{array}$} & \multirow[t]{5}{*}{ Setie eret } & \multirow[t]{5}{*}{ Aloaceae } & \multirow[t]{5}{*}{$\mathrm{H}$} & Eye disease & Latex & $\mathrm{F}$ & unprocessed plant & None \\
\hline & & & & Malaria & Leaves & $\mathrm{D}$ & Powdering & $\begin{array}{l}\text { Traditi } \\
\text { bevera }\end{array}$ \\
\hline & & & & Wound & Leaves & $\mathrm{F}$ & unprocessed plant & None \\
\hline & & & & Stomach-ache & Leaves & $\mathrm{F}$ & Crushing & $\begin{array}{l}\text { Traditi } \\
\text { bevera }\end{array}$ \\
\hline & & & & Snakebite & Leaves & $\mathrm{F}$ & unprocessed plant & None \\
\hline \multirow{2}{*}{$\begin{array}{l}\text { Entada } \\
\text { abyssinica A.Rich. }\end{array}$} & \multirow[t]{2}{*}{ Sheferie } & \multirow[t]{2}{*}{ Fabaceae } & \multirow[t]{2}{*}{$\mathrm{T}$} & Cellulitis & Leaves & $\mathrm{F}$ & Crushing & Vaseli \\
\hline & & & & Snakebite & Leaves & $\mathrm{F}$ & unprocessed plant & None \\
\hline Arundo donax L. & Shembeqo & Poaceae & $\mathrm{H}$ & Snakebite & Root & $\mathrm{F}$ & unprocessed root & None \\
\hline Moringa oleifera Lam. & Shiferaw & Moringaceae & $\mathrm{T}$ & Blood pressure & Leaves & $F / D$ & $\begin{array}{l}\text { Crushing and } \\
\text { boiling }\end{array}$ & Tea \\
\hline $\begin{array}{l}\text { Pennisetum } \\
\text { thunbergii Kunth }\end{array}$ & Sindedo & Poaceae & $\mathrm{H}$ & Rh-factor & Root & $\mathrm{D}$ & $\begin{array}{l}\text { Powdering and } \\
\text { baking with bread }\end{array}$ & None \\
\hline \multirow{2}{*}{$\begin{array}{l}\text { Rhus natalensis Bernh. } \\
\text { ex C.Krauss }\end{array}$} & \multirow[t]{2}{*}{ Taqima } & \multirow[t]{2}{*}{ Anacardiaceae } & \multirow[t]{2}{*}{$S$} & Scabies & Leaves & $\mathrm{F}$ & Crushing & Vaseli \\
\hline & & & & Cellulitis & Leaves & $\mathrm{F}$ & Crushing & Vaseli \\
\hline \multirow{5}{*}{$\begin{array}{l}\text { Jasminum } \\
\text { abyssinicum Hochets. } \\
\text { Ex DC. }\end{array}$} & \multirow[t]{5}{*}{ Tebelel } & \multirow[t]{5}{*}{ Oleaceae } & \multirow[t]{5}{*}{$S$} & Snakebite & Leaves & $\mathrm{F}$ & Crushing & Water \\
\hline & & & & Hemorroids & Stem & $\mathrm{F}$ & Heating & None \\
\hline & & & & Jaundice & Leaves & $\mathrm{F}$ & Crushing & Milk \\
\hline & & & & Taeniasis & Leaves & $\mathrm{F}$ & unprocessed plant & None \\
\hline & & & & Eye disease & Root & $\mathrm{F}$ & $\begin{array}{l}\text { Crushing and } \\
\text { squeezing }\end{array}$ & None \\
\hline $\begin{array}{l}\text { Eragrostis tef (Zucc.) } \\
\text { Trotter }\end{array}$ & Tef & Poaceae & $\mathrm{H}$ & Rabies & Seeds & D & Powdering & $\begin{array}{l}\text { Bakinc } \\
\text { sativu }\end{array}$ \\
\hline $\begin{array}{l}\text { Cymbopogon } \\
\text { citratus (DC.) Stapf }\end{array}$ & Tej sar & Poaceae & $\mathrm{H}$ & $\begin{array}{l}\text { "Mognbagegn" } \\
\text { (Anthrax) }\end{array}$ & Leaves & D & Powdering & Water \\
\hline Linum usitatissimum L. & Telba & Linaceae & $\mathrm{H}$ & Diarrhoea & Seeds & $\mathrm{D}$ & Powdering & Water \\
\hline & & & & Intestinal strangulation & Seeds & $\mathrm{D}$ & Powdering & Water \\
\hline Achyranthes aspera $L$ & Telenj & Amaranthaceae & $S$ & Wound & Leaves & $\mathrm{F}$ & Crushing & None \\
\hline Ruta chalepensis L. & Tenadam & Rutaceae & $\mathrm{H}$ & Abdominal pain & Leaves/fruits & $\mathrm{F}$ & unprocessed plant & Tea \\
\hline
\end{tabular}




\begin{tabular}{|c|c|c|c|c|c|c|c|c|}
\hline Scientific name & LN & Family & LF & DT & PU & $\mathrm{CU}$ & PM & IA \\
\hline \multirow[t]{2}{*}{ Symphytum officinale L. } & \multirow[t]{2}{*}{ Tenkul } & \multirow[t]{2}{*}{ Boraginaceae } & \multirow[t]{2}{*}{$\mathrm{H}$} & Eye disease & Leaves & $\mathrm{F}$ & Squeezing & None \\
\hline & & & & $\begin{array}{l}\text { Excessive bleeding of } \\
\text { menstruation }\end{array}$ & Leaves & $\mathrm{F}$ & Crushing & Water \\
\hline \multirow[t]{2}{*}{ Nicotiana tabacum L. } & \multirow[t]{2}{*}{ Tinbaho } & \multirow[t]{2}{*}{ Solanaceae } & \multirow[t]{2}{*}{$\mathrm{H}$} & Snakebite & Leaves & $\mathrm{F}$ & Crushing & Water \\
\hline & & & & Leech & Leaves & $\mathrm{F}$ & Crushing & Water \\
\hline \multirow[t]{3}{*}{$\begin{array}{l}\text { Otostegia integrifolia } \\
\text { Benth. }\end{array}$} & \multirow[t]{3}{*}{ Tinjut } & \multirow[t]{3}{*}{ Lamiaceae } & \multirow[t]{3}{*}{$S$} & Evil eye & Leaves & D & $\begin{array}{l}\text { Powdering and } \\
\text { smoking }\end{array}$ & None \\
\hline & & & & $\begin{array}{l}\text { “Megagna” (unidentified } \\
\text { gastrointestinal } \\
\text { disorder) }\end{array}$ & Leaves & D & $\begin{array}{l}\text { Powdering and } \\
\text { smoking }\end{array}$ & None \\
\hline & & & & Snakebite & Leaves & $\mathrm{F}$ & Crushing & Water \\
\hline Euphorbia cactus Boiss. & $\begin{array}{l}\text { Tinshuwa } \\
\text { qoleqol }\end{array}$ & Euphorbiaceae & $S$ & Leishmaniasis & Latex & $\mathrm{F}$ & Squeezing & $\begin{array}{l}\text { Mixinc } \\
\text { dough }\end{array}$ \\
\hline $\begin{array}{l}\text { Maytenus arbutifolia (A. } \\
\text { Rich.) Wilczek }\end{array}$ & Tiqur atat & Celastraceae & $S$ & Abdominal pain & Stem bark & $\mathrm{F}$ & unprocessed plant & None \\
\hline $\begin{array}{l}\text { Solanum nigrum Acerbi } \\
\text { ex Dunal }\end{array}$ & Tiqur awut & Solanaceae & $\mathrm{H}$ & Wound & Leaves & $\mathrm{F}$ & Crushing & None \\
\hline Nigella sativa L. & Tiqur azmud & Ranunculaceae & $\mathrm{H}$ & Cough & Seeds & D & Powdering & $\begin{array}{l}\text { Boiling } \\
\text { butter }\end{array}$ \\
\hline $\begin{array}{l}\text { Prunus africana Hook. f. } \\
\text { Kalkm. }\end{array}$ & Tiqur enchet & Podocarpaceae & $\mathrm{T}$ & Evil eye & Root & $\mathrm{D}$ & $\begin{array}{l}\text { Powdering and } \\
\text { smoking }\end{array}$ & None \\
\hline Cordia Africana Lam. & Wanza & Boraginaceae & $\mathrm{T}$ & Fire burn & Leaves & $\mathrm{D}$ & Crushing & Vaseli \\
\hline Ficus vasta Forssk & Warka & Moraceae & $\mathrm{T}$ & Evil eye & Stem bark & $\mathrm{D}$ & $\begin{array}{l}\text { Powdering and } \\
\text { smoking }\end{array}$ & None \\
\hline $\begin{array}{l}\text { Silene macrosolen A. } \\
\text { Rich. }\end{array}$ & Wogert & Caryophyllaceae & $\mathrm{H}$ & Evil eye & Root & $\mathrm{D}$ & $\begin{array}{l}\text { Powdering and } \\
\text { smoking }\end{array}$ & None \\
\hline \multirow{2}{*}{$\begin{array}{l}\text { Olea europaea L. subsp. } \\
\text { cuspidata (Wall. ex } \\
\text { G.Don) Cif. }\end{array}$} & \multirow[t]{2}{*}{ Woira } & \multirow[t]{2}{*}{ Oleaceae } & \multirow[t]{2}{*}{$\mathrm{T}$} & Hemorroids & Leaves & $\mathrm{F}$ & Crushing & None \\
\hline & & & & Vomiting & Leaves & $\mathrm{F}$ & Crushing & $\begin{array}{l}\text { Mixinc } \\
\text { with } L \\
\text { sativu } \\
\text { water }\end{array}$ \\
\hline $\begin{array}{l}\text { Citrus } \\
\text { aurantiifolia (Christm.) } \\
\text { Swingle }\end{array}$ & Yabesha lomi & Rutaceae & S & Cellulitis & Root & $\mathrm{F}$ & Crushing & None \\
\hline $\begin{array}{l}\text { Juniperus } \\
\text { procera Hochst. ex Endl. }\end{array}$ & Yabesha tid & Cupressaceae & $\mathrm{T}$ & Vomiting & Leaves & $\mathrm{F}$ & Crushing & Tea \\
\hline \multirow{5}{*}{$\begin{array}{l}\text { Verbascum } \\
\text { sinaiticum Benth. }\end{array}$} & \multirow[t]{5}{*}{ Yahya joro } & \multirow[t]{5}{*}{ Scrophulariaceae } & \multirow[t]{5}{*}{$\mathrm{H}$} & Snakebite & Root & $\mathrm{F}$ & unprocessed plant & None \\
\hline & & & & Evil eye & Root & $\mathrm{D}$ & $\begin{array}{l}\text { Powdering and } \\
\text { smoking }\end{array}$ & None \\
\hline & & & & Wound & Root & $\mathrm{F}$ & Crushing & Water \\
\hline & & & & Tropical ulcer & Root & $\mathrm{F}$ & Crushing & Water \\
\hline & & & & Wart & Leaves & $\mathrm{F}$ & Heating & None \\
\hline Impans tinctoria A.Rich. & $\begin{array}{l}\text { Yebereha } \\
\text { shimbra }\end{array}$ & Balsaminaceae & $\mathrm{H}$ & Snakebite & Root & $\mathrm{F}$ & Unprocessed plant & None \\
\hline $\begin{array}{l}\text { Brucea } \\
\text { antidysenterica J.F.Mill. }\end{array}$ & Yenebir tifir & Simaroubaceae & S & Leishmaniasis & Seeds & $\mathrm{D}$ & Powdering & $\begin{array}{l}\text { Bakins } \\
\text { dough }\end{array}$ \\
\hline \multirow[t]{2}{*}{ Xanthium oriental L. } & \multirow[t]{2}{*}{ Yejema qitel } & \multirow[t]{2}{*}{ Asteraceae } & \multirow[t]{2}{*}{$\mathrm{H}$} & Wound & Leaves & $\mathrm{D}$ & Powdering & Butter \\
\hline & & & & Scabies & Leaves & $\mathrm{D}$ & Powdering & Butter \\
\hline $\begin{array}{l}\text { Commicarpus } \\
\text { plumbagineus (Cav.) } \\
\text { Standl. }\end{array}$ & Yejib chama & Nyctaginaceae & $\mathrm{H}$ & Snakebite & Leaves & $\mathrm{F}$ & Unprocessed plant & None \\
\hline \multirow{2}{*}{$\begin{array}{l}\text { Cucumis ficifolius A. } \\
\text { Rich }\end{array}$} & \multirow[t]{2}{*}{ Yemdir embuay } & \multirow[t]{2}{*}{ Cucurbitaceae } & \multirow[t]{2}{*}{ C } & Abdominal pain & Root & $\mathrm{F}$ & Crushing & Water \\
\hline & & & & Swelling on the body & Root & $\mathrm{F}$ & Crushing & Water \\
\hline
\end{tabular}

Page $11 / 27$ 


\begin{tabular}{|c|c|c|c|c|c|c|c|c|}
\hline Scientific name & LN & Family & LF & DT & PU & $\mathrm{CU}$ & PM & IA \\
\hline & & & & Snakebite & Root & $\mathrm{F}$ & unprocessed plant & None \\
\hline & & & & Rickets & Root & $\mathrm{F}$ & Crushing & None \\
\hline & & & & Infant dysentery & Root & $\mathrm{F}$ & Crushing & None \\
\hline & & & & Vomiting & Root & $\mathrm{F}$ & unprocessed plant & None \\
\hline & & & & Retained placenta & Root & $\mathrm{F}$ & Crushing & None \\
\hline & & & & Cellulitis & Root & $\mathrm{F}$ & Crushing & Water \\
\hline $\begin{array}{l}\text { Plectranthus } \\
\text { amboinicus (Lour.) } \\
\text { Spreng. }\end{array}$ & $\begin{array}{l}\text { Yequsil } \\
\text { medhanit }\end{array}$ & Lamiaceae & $\mathrm{H}$ & Wound & Leaves & $\mathrm{F}$ & Crushing & None \\
\hline Berberis holstii Engl. & Yeset af & Berberidaceae & S & Evil eye & Leaves & D & $\begin{array}{l}\text { Powdering and } \\
\text { smoking }\end{array}$ & None \\
\hline $\begin{array}{l}\text { Solanecio gigas (Vatke) } \\
\text { C. Jeffrey }\end{array}$ & $\begin{array}{l}\text { Yeshikoko } \\
\text { gomen }\end{array}$ & Asteraceae & S & Diarrhoea & Leaves & $\mathrm{F}$ & Crushing & None \\
\hline $\begin{array}{l}\text { Sorghum } \\
\text { bicolor Moench }\end{array}$ & Zengada & Poaceae & $\mathrm{H}$ & Diarrhoea & Seeds & D & Powdering & $\begin{array}{l}\text { Boilinc } \\
\text { coffee }\end{array}$ \\
\hline \multirow[t]{3}{*}{ Solanum anguivi Lam. } & \multirow[t]{3}{*}{ Zerch embuay } & \multirow[t]{3}{*}{ Solanaceae } & \multirow[t]{3}{*}{ s } & Blemish & Leaves & D & Powdering & Vaseli \\
\hline & & & & Wound & Fruit & $\mathrm{F}$ & Crushing & None \\
\hline & & & & Impotence & Root & $\mathrm{F}$ & Crushing & $\begin{array}{l}\text { Traditi } \\
\text { bevera }\end{array}$ \\
\hline $\begin{array}{l}\text { Zingiber } \\
\text { officinale Roscoe }\end{array}$ & Zinjibil & Zingiberaceae & $\mathrm{H}$ & Cough & Root & $\mathrm{F} / \mathrm{D}$ & $\begin{array}{l}\text { Crushing and } \\
\text { boiling }\end{array}$ & Water \\
\hline \multirow[t]{3}{*}{ Satureja punctata R.Br. } & \multirow{3}{*}{$\begin{array}{l}\text { Este- } \\
\text { meaza/lomi } \\
\text { kesie }\end{array}$} & \multirow[t]{3}{*}{ Lamiaceae } & \multirow[t]{3}{*}{$\mathrm{H}$} & Snakebite & Root & $\mathrm{F}$ & unprocessed plant & None \\
\hline & & & & Diarrhoea & Root & $\mathrm{F}$ & unprocessed plant & None \\
\hline & & & & Evil eye & Whole part & D & $\begin{array}{l}\text { Powdering and } \\
\text { smoking }\end{array}$ & None \\
\hline $\begin{array}{l}\text { Asparagus } \\
\text { africanus Lam. }\end{array}$ & Seriti & Asparagaceae & S & Impotence & Root & D & Powdering & $\begin{array}{l}\text { Traditi } \\
\text { bevera }\end{array}$ \\
\hline $\begin{array}{l}\text { Chenopodium } \\
\text { ambrosioides L. }\end{array}$ & Sinign & Amaranthaceae & $\mathrm{H}$ & "Mognbagegn"(Anthrax) & Leaves & D & Powdering & Water \\
\hline Carica papaya L. & Papaya & Caricaceae & s & Ascariasis & Seeds & D & Powdering & Water \\
\hline $\begin{array}{l}\text { Guizotia } \\
\text { abyssinica (L.f.) Cass. }\end{array}$ & Nug & Asteraceae & $\mathrm{H}$ & Cough & Seeds & D & Powdering & $\begin{array}{l}\text { Boilinc } \\
\text { butter }\end{array}$ \\
\hline Lens culinaris Medik. & Misir & Fabaceae & $\mathrm{H}$ & Herpes zoster & Seeds & D & Roasting in water & None \\
\hline $\begin{array}{l}\text { Veronica anagallis- } \\
\text { aquatica L. }\end{array}$ & $\begin{array}{l}\text { Leteqatele } \\
\text { sewunet }\end{array}$ & Scrophulariaceae & $\mathrm{H}$ & Fire burn & Whole part & $\mathrm{F}$ & Squeezing & None \\
\hline \multirow{2}{*}{$\begin{array}{l}\text { Rhamnus } \\
\text { prinoides L'Hér. }\end{array}$} & \multirow[t]{2}{*}{ Gesho } & \multirow[t]{2}{*}{ Rhamnaceae } & \multirow[t]{2}{*}{$s$} & Scabies & Seeds & D & Powdering & Vaseli \\
\hline & & & & Rickets & Shoot tips & $\mathrm{F}$ & Crushing & $\begin{array}{l}\text { Mixinc } \\
\text { Leave: } \\
\text { Verno } \\
\text { amygc } \\
\text { mixing } \\
\text { wate }\end{array}$ \\
\hline Lippia adoenis Hochst. & Yelam kessie & Verbenaceae & s & Eye diseases & Leaves & $\mathrm{F}$ & Crushing & Water \\
\hline $\begin{array}{l}\text { Debregeasia } \\
\text { saeneb (Forssk.) Hepper } \\
\text { \& Wood }\end{array}$ & Wonz admiq & Urticaceae & S & Jaundice & Leaves & $\mathrm{F}$ & Crushing & $\begin{array}{l}\text { Bakinc } \\
\text { injera }\end{array}$ \\
\hline $\begin{array}{l}\text { Heteromorpha } \\
\text { arborescens (Spreng.) } \\
\text { Cham. \& Schltdl. }\end{array}$ & Yejib mirkuz & Apiaceae & s & Diarrhoea & Leaves & $\mathrm{F}$ & Crushing & Water \\
\hline \multirow[t]{2}{*}{ Salix mucronata Thunb. } & \multirow[t]{2}{*}{ Akeya } & \multirow[t]{2}{*}{ Salicaceae } & \multirow[t]{2}{*}{$\mathrm{s}$} & Tooth-ache & Stem & $\mathrm{F}$ & Unprocessed plant & None \\
\hline & & & & Rabies & Leaves & $\mathrm{F}$ & Crushing & Water \\
\hline
\end{tabular}




\begin{tabular}{|c|c|c|c|c|c|c|c|c|}
\hline Scientific name & $\mathrm{LN}$ & Family & LF & DT & PU & $\mathrm{CU}$ & PM & IA \\
\hline $\begin{array}{l}\text { Pterolobium } \\
\text { stellatum (Forssk.) } \\
\text { Brenan }\end{array}$ & Qentefa & Fabaceae & $S$ & Circumcision wound & Leaves & $\mathrm{F}$ & Crushing & None \\
\hline $\begin{array}{l}\text { Rubus } \\
\text { steudneri Schweinf. }\end{array}$ & Enjori & Rosaceae & $S$ & Swelling on the body & $\begin{array}{l}\text { Leaves and } \\
\text { fruits }\end{array}$ & $\mathrm{F}$ & Crushing & None \\
\hline Scorpiurus muricatus L & Worteb & Fabaceae & $\mathrm{H}$ & Wound & Leaves & $\mathrm{F}$ & Crushing & None \\
\hline
\end{tabular}

\begin{tabular}{|c|c|c|c|}
\hline No. & Families & Number of species & Percentage \\
\hline 1 & Fabaceae & 14 & 9.9 \\
\hline 2 & Lamiaceae & 9 & 6.3 \\
\hline 3 & Solanaceae & 9 & 6.3 \\
\hline 4 & Asteraceae & 8 & 5.6 \\
\hline 5 & Euphorbiaceae & 8 & 5.6 \\
\hline 6 & Poaceae & 8 & 5.6 \\
\hline 7 & Apiaceae & 4 & 2.8 \\
\hline 8 & Cucurbitaceae & 4 & 2.8 \\
\hline 9 & Malvaceae & 4 & 2.8 \\
\hline 10 & Verbenaceae & 4 & 2.8 \\
\hline 11 & Amaranthaceae & 3 & 2.1 \\
\hline 12 & Apocynaceae & 3 & 2.1 \\
\hline 13 & Celastraceae & 3 & 2.1 \\
\hline 14 & Polygonaceae & 3 & 2.1 \\
\hline 15 & Urticaceae & 3 & 2.1 \\
\hline 16 & Amaryllidaceae & 2 & 1.4 \\
\hline 17 & Anacardiaceae & 2 & 1.4 \\
\hline 18 & Boraginaceae & 2 & 1.4 \\
\hline 19 & Crassulaceae & 2 & 1.4 \\
\hline 20 & Myrsinaceae & 2 & 1.4 \\
\hline 21 & Myrtaceae & 2 & 1.4 \\
\hline 22 & Oleaceae & 2 & 1.4 \\
\hline 23 & Polygalaceae & 2 & 1.4 \\
\hline 24 & Ranunculaceae & 2 & 1.4 \\
\hline 25 & Rhamnaceae & 2 & 1.4 \\
\hline 26 & Rubiaceae & 2 & 1.4 \\
\hline 27 & Rutaceae & 2 & 1.4 \\
\hline 28 & Scrophulariaceae & 2 & 1.4 \\
\hline 29 & Vitaceae & 2 & 1.4 \\
\hline \multirow[t]{2}{*}{30} & The rest & 27 & 19 \\
\hline & Total & 142 & 100.0 \\
\hline
\end{tabular}

\subsection{Life forms and habitats of medicinal plants}

The majority of medicinal plants (122) were collected from wild habitats (85.9\%) and with herbs accounted (42.3\%) followed by shrubs (41.5\%), trees (11.3\%), and least represented by climbers (6\%). The rest of the 20 medicinal plants were cultivated in the home garden (Figure 2).

\subsection{Parts of medicinal plants used for remedy preparation}

Page $13 / 27$ 
The study revealed that diverse types of plant parts were used to treat various ailments either in combination or alone. The analysis of collected information showed that leaves were the widely used plant parts that accounted for 119 (45.4\%) followed by root 74 (28.2), Seed (6.9) and the rest all accounted $19.5 \%$, epiphytes, and whole parts are the least used for remedy preparation (Table 3 ).

Table 3.Medicinal plant parts used to prepare traditional medicines in Ensaro district

\begin{tabular}{|llll|}
\hline No & Parts used & Frequency & Percentage \\
\hline 1 & Leaves & 119 & 45.4 \\
\hline 2 & Root & 74 & 28.2 \\
\hline 3 & Seeds & 18 & 6.9 \\
\hline 4 & Stem bark & 14 & 5.3 \\
\hline 5 & Bulb & 8 & 3.1 \\
\hline 6 & Fruit & 6 & 2.3 \\
\hline 7 & Whole part & 5 & 1.9 \\
\hline 8 & Latex & 4 & 1.5 \\
\hline 9 & Shoot tips & 3 & 1.1 \\
\hline 10 & Stem juice & 3 & 1.1 \\
\hline 11 & Rhizome & 2 & 0.8 \\
\hline 12 & Root bark & 2 & 0.8 \\
\hline 13 & Stem & 2 & 0.8 \\
\hline 14 & Aerial part & 1 & 0.4 \\
\hline 15 & Epiphyte & 1 & 0.4 \\
\hline & Total & 262 & 100.0 \\
\hline
\end{tabular}

\subsection{Plant conditions for preparation}

Medicines were prepared in fresh, dry, or both dry and fresh conditions of plant parts. The Marjory of traditional herbal medicines prepared in fresh form (63\%), followed by dry form (30\%) (Figure 3 ).

\subsection{Methods of medicine preparation}

Table 4 Depicts the different types of traditional remedy preparations from medicinal plant parts. The main preparation method was crushing of the fresh or dried plant materials that accounted for $43.6 \%$, followed by unprocessed plant $17.4 \%$ and powdering $(16.2 \%)$.

Table 4. Traditional healers use different ways to prepare medicines from medicinal plants 


\begin{tabular}{llll} 
No & Preparation methods & Frequency & Percentage \\
\hline 1 & Crushing & 113 & 43.6 \\
\hline 2 & Unprocessed & 45 & 17.4 \\
\hline 3 & Powdering & 42 & 16.2 \\
\hline 4 & Powdering and smoking & 27 & 10.4 \\
\hline 5 & Crushing and squeezing & 6 & 2.3 \\
\hline 6 & Heating & 5 & 1.9 \\
\hline 7 & Powdering and boiling & 5 & 1.9 \\
\hline 8 & Boiling & 4 & 1.5 \\
\hline 9 & Powdering and baking & 4 & 1.5 \\
\hline 10 & Squeezing & 4 & 1.5 \\
\hline 11 & Roasting & 2 & 0.4 \\
\hline 12 & Heating and Powdering & 1 & 0.4 \\
\hline 13 & Powdering and soaking in water & 1 & 259 \\
\hline & Total & & 10.0 \\
\hline
\end{tabular}

\subsection{Methods of treatments}

This study reported a wide range of traditional remedy applications. Smearing was the most common, accounting for 85 (32.9 percent), followed by drinking (26.0 percent) (Table 5)

Table 5.Methods of applications

\begin{tabular}{|c|c|c|c|}
\hline No & Methods of applications & Frequency & Percentage \\
\hline 1. & Smearing & 85 & 32.9 \\
\hline 1. & Drinking & 67 & 26.0 \\
\hline 1. & Chewing & 33 & 12.8 \\
\hline 1. & Inhaling & 32 & 12.4 \\
\hline 1. & Eating & 16 & 6.2 \\
\hline 1. & Wrapping & 10 & 3.9 \\
\hline 1. & dropping & 8 & 3.1 \\
\hline 1. & Washing & 3 & 1.2 \\
\hline 1. & Heating & 2 & 0.8 \\
\hline 1. & Holding on teeth & 1 & 0.4 \\
\hline 1. & Sniffing & 1 & 0.4 \\
\hline Total & & 258 & 100.0 \\
\hline
\end{tabular}

\subsection{Routes of administration and dosage}

The current study indicated the different routes for the applications of traditional herbal medicines for the treatment of various types of human ailments and diseases. Oral administration is the most used route 117(45.3\%) which followed by the dermal application while the optical route is the least used organ 101 (39.1\%) Figure 4. 
3.9 Human diseases treated by medicinal plants in the study area

In the present study district, 52 human diseases being treated by traditional herbal medicines. Table 6 indicates that most ailments are treated by more than one medicinal plant species. For example, cellulitis (contact dermatitis) treated by Dichrostachys cinerea, Premna schimperii, Zehneria scabra, Maytenus senegalensis and Rhus natalensis.

Table 6. Human diseases treated by medicinal plants in Ensaro district 


\begin{tabular}{|c|c|c|c|}
\hline No & Ailments & Frequency & $\begin{array}{l}\text { Number of medicinal plants } \\
\text { used to treat }\end{array}$ \\
\hline 1. & Cellulitis & 90 & 18 \\
\hline 1. & Snakebite & 79 & 23 \\
\hline 1. & Evil eye & 77 & 21 \\
\hline 1. & Scabies & 63 & 7 \\
\hline 1. & "Likft"(devil illness) & 56 & 3 \\
\hline 1. & Mitch & 47 & 8 \\
\hline 1. & Scorpion bite & 43 & 3 \\
\hline 1. & Herpes zoster & 41 & 2 \\
\hline 1. & Leishmaniasis & 33 & 3 \\
\hline 1. & Epilepsy & 31 & 1 \\
\hline 1. & Dandruff & 30 & 4 \\
\hline 1. & Taeniasis & 29 & 4 \\
\hline 1. & Abdominal pain & 24 & 10 \\
\hline 1. & Wound & 23 & 25 \\
\hline 1. & Eczema & 23 & 5 \\
\hline 1. & Diarrhea & 21 & 11 \\
\hline 1. & Blemish & 20 & 1 \\
\hline 1. & Jaundice & 19 & 11 \\
\hline 1. & $\begin{array}{l}\text { "Megagna" (unidentified } \\
\text { gastrointestinal disorder) }\end{array}$ & 19 & 6 \\
\hline 1. & Dysentery in infants & 17 & 3 \\
\hline 1. & Tumor & 15 & 4 \\
\hline 1. & Delayed placenta & 15 & 2 \\
\hline 1. & Retained placenta & 15 & 2 \\
\hline 1. & Rickets & 13 & 2 \\
\hline 1. & Hemorrhoids & 9 & 6 \\
\hline 1. & Intestinal strangulation & 8 & 1 \\
\hline 1. & Rabies & 7 & 6 \\
\hline 1. & Cough & 7 & 4 \\
\hline
\end{tabular}




\begin{tabular}{|c|c|c|c|}
\hline No & Ailments & Frequency & $\begin{array}{l}\text { Number of medicinal plants } \\
\text { used to treat }\end{array}$ \\
\hline 1. & Tonsillitis & 7 & 3 \\
\hline 1. & Impotence & 6 & 4 \\
\hline 1. & Circumcise infection & 6 & 3 \\
\hline 1. & Stomach-ache & 6 & 2 \\
\hline 1. & Bleeding & 6 & 1 \\
\hline 1. & Rh-factor & 5 & 2 \\
\hline 1. & Abortion & 5 & 1 \\
\hline 1. & “Mognbagegn” (Anthrax) & 4 & 5 \\
\hline 1. & Dog bite & 4 & 1 \\
\hline 1. & Blood pressure & 3 & 6 \\
\hline 1. & $\begin{array}{l}\text { Excessive bleeding of } \\
\text { menstruation }\end{array}$ & 3 & 4 \\
\hline 1. & Malaria & 3 & 3 \\
\hline 1. & Wart & 3 & 2 \\
\hline 1. & Leech & 3 & 1 \\
\hline 1. & Eye disease & 2 & 7 \\
\hline 1. & Typhoid & 2 & 2 \\
\hline 1. & Asthma & 2 & 1 \\
\hline 1. & Leprosy & 2 & 1 \\
\hline 1. & Syphilis & 2 & 1 \\
\hline 1. & Fire burn & 1 & 3 \\
\hline 1. & Vomiting & 1 & 3 \\
\hline 1. & Ascariasis & 1 & 2 \\
\hline 1. & Tooth-ache & 1 & 1 \\
\hline 1. & Tropical ulcer & 1 & 1 \\
\hline
\end{tabular}

\section{Preference ranking}

The five most reported medicinal plants for effective treatment of cellulitis that reported frequently in the study area were selected for preference ranking exercise. Ten key informants were asked to rank the given medicinal plants based on their usefulness. They were also asked to give numbers 5 for effective and 1 for the less effective medicinal plants. As the result, Dichrostachys cinerea was ranked first and Zehneria scabra ranked second whereas Rhus natalensis was ranked last ( 
Table 7).

Table 7. Preference ranking of medicinal plants used for the treatment of cellulitis (skin contact dermatitis)

\begin{tabular}{|c|c|c|c|c|c|c|c|c|c|c|c|c|}
\hline \multirow[t]{2}{*}{ Name of medicinal plants } & \multicolumn{10}{|c|}{ Informants from (A-J) } & \multirow[t]{2}{*}{ Total } & \multirow[t]{2}{*}{ Rank } \\
\hline & $A$ & B & $\mathrm{C}$ & D & $\mathrm{E}$ & $\mathrm{F}$ & G & $\mathrm{H}$ & I & $\mathrm{J}$ & & \\
\hline Dichrostachys cinerea (L.) Wight \& Arn. & 4 & 5 & 4 & 5 & 5 & 4 & 4 & 5 & 5 & 5 & 46 & $1^{\text {st }}$ \\
\hline Clematis simensis Fresen. & 3 & 5 & 3 & 4 & 2 & 1 & 3 & 2 & 1 & 2 & 26 & $4^{\text {th }}$ \\
\hline Premna schimperii Engl. & 5 & 3 & 2 & 4 & 3 & 3 & 4 & 5 & 2 & 3 & 34 & $3^{\text {rd }}$ \\
\hline Zehneria scabra Sond. & 5 & 3 & 4 & 4 & 3 & 2 & 1 & 4 & 5 & 4 & 35 & $2^{\text {nd }}$ \\
\hline Rhus natalensis Bernh. & 2 & 4 & 2 & 3 & 2 & 1 & 2 & 3 & 3 & 3 & 25 & $5^{\text {th }}$ \\
\hline
\end{tabular}

\section{Fidelity level}

To identify the most preferred medicinal plant species used to treat certain ailments in the study area. Hence, Ocimum lamiifolium, Albizia anthelmintica, Phytolacca dodecandra, Dichrostachys cinerea, Andrachne aspera Spreng, Psydrax schimperiana, Emilia sonchifolia, Clematis simensis, Calpurnia aurea, Silene macrosolen and Securidaca longipedunculata Fresen had the highest fidelity level values that used as a sign of their healing potential in the study area (Table 8 ).

Table 8. The relative healing potential of the 14 most cited medicinal plants used against human diseases

\begin{tabular}{|lllll|}
\hline Disease categories & Species & Ip & lu & Fidelity level \\
\hline Mitch & Ocimum lamiifolium Hochst. ex Benth. & 6 & 6 & 100 \\
\hline Taeniasis & Albizia anthelmintica Brongn. & 23 & 23 & 100 \\
\hline Rabies & Phytolacca dodecandra L' Her. & 26 & 27 & 96.3 \\
\hline Cellulitis & Dichrostachys cinerea (L.) Wight \& Arn. & 21 & 22 & 95.5 \\
\hline Snakebite & Andrachne aspera Spreng & 17 & 18 & 94.4 \\
\hline Impotence & Emilia sonchifolia (L.) DC. ex DC. & 12 & 14 & 85.7 \\
\hline Haemorrhoids & Clematis simensis Fresen. & 21 & 25 & 84 \\
\hline Jaundice & Calpurnia aurea (Ait.) Benth. & 31 & 37 & 83.8 \\
\hline Likfit & Silene macrosolen Steud. ex A. Rich. & 15 & 18 & 83.3 \\
\hline Evil eye & Securidaca longipedunculata Fresen & 12 & 15 & 80 \\
\hline Diarrhoea & Cucumis ficifolius A. Rich. & 19 & 25 & 76 \\
\hline Tonsillitis & Salvia nilotica Jacq. & 38 & 50 & 76 \\
\hline Wound & Achyranthes aspera L. var. procera Fiori & 13 & 18 & 72.2 \\
\hline
\end{tabular}

\section{Direct matrix ranking}

In addition to food values, the local people used the plants for other different purposes such as fuelwood, fence, house construction, medicine, farm implements, and furniture. The result of direct matrix ranking revealed that Cordia Africana, Ziziphus spina-christi, Olea europaea subsp. cuspidata, Juniperus procera, Millettia ferruginea, Eucalyptus globulus, Rubus apetalus, Carissa spinarum, Dichrostachys cinerea were ranked first to ninth, respectively. Similarly, the seven use-values reported on six selected plant species were summed up and ranked and, the result showed that fuelwood collection, fence, farm implements, house construction, medicine, fodder, food were ranked first, second, third, fourth, fifth, sixth and seventh, respectively (Table 9).

Table 9.Direct matrix ranking of six plant species by twelve informants based on seven use criteria (5= best; $4=$ very good; $3=$ good; $2=$ less used; $1=1$ east used and $0=$ no value) 


\begin{tabular}{|c|c|c|c|c|c|c|c|c|c|}
\hline \multirow[t]{2}{*}{ Species } & \multicolumn{9}{|c|}{ Use diversity } \\
\hline & Medicine & Fuelwood & Fence & Farm implements & House construction & Fodder & Food & total & Rank \\
\hline Dichrostachys cinerea & 3 & 2 & 4 & 1 & 1 & 1 & 0 & 12 & $9^{\text {th }}$ \\
\hline Carissa spinarum & 3 & 1 & 4 & 0 & 0 & 2 & 3 & 13 & $8^{\text {th }}$ \\
\hline Millettia ferruginea & 3 & 3 & 1 & 5 & 5 & 1 & 0 & 18 & $5^{\text {th }}$ \\
\hline Rubus apetalus & 2 & 3 & 2 & 0 & 0 & 4 & 3 & 14 & $7^{\text {th }}$ \\
\hline Ziziphus spina-christi & 3 & 4 & 5 & 4 & 1 & 3 & 4 & 25 & $2^{\text {nd }}$ \\
\hline Eucalyptus globulus & 1 & 4 & 1 & 2 & 3 & 0 & 0 & 11 & $6^{\text {th }}$ \\
\hline Cordia Africana & 3 & 4 & 4 & 5 & 5 & 3 & 3 & 27 & $1^{\text {st }}$ \\
\hline Olea europaea subsp. cuspidata & 4 & 5 & 3 & 5 & 5 & 0 & 0 & 22 & $3^{\text {rd }}$ \\
\hline Juniperus procera & 1 & 5 & 3 & 5 & 5 & 0 & 0 & 19 & $4^{\text {th }}$ \\
\hline Total & 23 & 30 & 28 & 27 & 25 & 14 & 13 & & \\
\hline Rank & $5^{\text {th }}$ & $1^{\text {st }}$ & $2^{\text {nd }}$ & $3 r d$ & $4^{\text {th }}$ & $6^{\text {th }}$ & $7^{\text {th }}$ & & \\
\hline
\end{tabular}

\section{Discussion}

\subsection{Medicinal plant species composition}

The present study showed that Ensaro district has a relatively high diversity of medicinal plants. this high diversity of plant species may be related to the existence of different topography and climatic conditions of the study area. According to Asefa et al. (2020), the presence of high plant diversity in Ethiopia is mainly linked with the existence of different topography and climate.

Although the modern health center is widely distributed and accessible to the inhabitants of the study area, the results of this study demonstrated that indigenous knowledge of using medicinal plants is still rich now. On the one hand, this could be related to a cultural attitude regarding medicinal plant efficacy (Lulekal et al., 2013) while on the other hand, it could be due to a lack of or unaffordable pricing of modern pharmaceuticals (Zerabruk \& Yirga, 2012).

The number of medicinal plants collected from the current study area is comparable with study results reported in different parts of Ethiopia (Lulekal et al., 2013; Fenetahun et al., 2017; Kebede et al., 2018; Kelemu \& Wolde, 2018; Yohannis et al., 2018). Even though most medicinal plants recorded in the present study were mentioned by several authors in the country (Teklehaymanot et al., 2007; Yirga et al., 2012; d'Avigdor et al., 2014; Alemayehu et al., 2015), few medicinal plant species (Polygala abyssinica, Otostegia fruticosea, Psydrax schimperiana,) reported in the present study were not reported as medicine elsewhere in Ethiopia.

Seven medicinal plant species of this study such as Aloe pulcherrima Gilbert \& Sebsebe, Echinops kebericho Mesfin, Millettia ferruginea (Hochst.) Bak., Solanecio gigas (Vatke) C. Jeffrey, Taverniera abyssinica A. Rich., Urtica simensis Steudel, and Eragrostis tef (Zucc.) Trotter were identified as endemic plants to Ethiopia (Awas, 2009) while Inula confertiflora, Taverniera abyssinica, Satureja punctata, Solanecio gigas, Lippia adoensi and Millettia ferruginea are red list medicinal plants (Vivero et al., 2005) .

In this study, in agreement with the investigation conducted in Ankober district (Lulekal et al., 2013), Kilte Awulaelo District (Teklay et al., 2013), and Libo Kemkem District (Chekole et al., 2015) of Ethiopia, the dominant medicinal plant families documented were Fabaceae, Lamiaceae, Solanaceae,

Euphorbiaceae, Asteraceae, and Poaceae. This could be related to the fact that these families are the most prevalent in Ethiopian and Eritrean flora (Tesfaye et al., 2020). Furthermore, recent chemical screening investigations revealed that the greatest number of plant species in these families have bioactive chemicals having a strong physiological impact against diseases causing microorganisms (Gazzaneo et al., 2005; Gebrelibanos et al., 2008).

\subsection{Plant life forms, parts used, mode of preparation and administration}

Many medicinal plants used in this study area were herbs. This could be due to the reason that herbs are more readily available in the nearby areas than trees and shrubs, which are usually picked from distant forests (Lulekal et al., 2013). According to Hailemariam et al. (2009), herbaceous plants are found more abundantly than other life forms of plants, especially during the wet season. This finding is aligned with most ethnobotanical studies of medicinal plants in Ethiopia (Teklehaymanot \& Giday, 2007; Mesfin et al., 2009; Lulekal et al., 2013).

The wild habitats of the Ensaro district were identified as the major reservoir of plants providing approximately $86 \%$ of reported medicinal plants. However, the study showed that these habitats are exposed to anthropogenic influences, which is a result of the ever-increasing human interference, leading to the threatening of numerous wild medicinal plants along with associated indigenous practices. This finding is consistent with the findings of several ethnobotanical studies conducted in Ethiopia (Birhanu, Abera, et al., 2015; Fenetahun et al., 2017; Tuasha et al., 2018; Tefera \& Yihune, 2019), in Karnataka (Prashanth Kumar \& Shiddamallayya, 2016), Iran (Hosseini et al., 2021) and Sudan (Adam et al., 2020). This indicated that the effort attempted to cultivate medicinal plants in the home garden is very low in many countries. 
Leaves are the most used plant part for the preparation of traditional herbal remedies in the study district. Many ethnobotanical investigations carried out in different parts of Ethiopia documented that leaves are the mainly used part (Teklehaymanot \& Giday, 2007; Tefera \& Yihune, 2019). This could be because the leaves of most plant species produce secondary metabolites especially flavonoids that can induce the desired biological action (Agisho et al., 2014; Yang et al., 2020). Moreover, most studies have recommended the use of leaves rather than roots, because utilizing roots results in the extinction of medicinal plant species permanently (Kiringe, 2006; Pascaline et al., 2011; Chowdhury et al., 2015).

Results of this study also showed that freshly harvested parts were extensively used in the preparation of traditional remedies to treat various ailments. Several recent studies showed that fresh parts have been used for remedial preparations (Lulekal et al., 2013; Chekole, 2017; Tuasha et al., 2018). The preference of freshly harvested plant materials to dry by traditional healers might be due to the presence of a high concentration of bioactive secondary metabolites in the fresh parts than dry parts (Lulekal et al., 2013; Agisho et al., 2014).

In the current study, Crushing is reported as the main remedies preparation method which is seen similarly in other ethnobotanical studies (Kidane et al., 2018; Abebe \& Chane Teferi, 2021; Assen et al., 2021). This is probably since crushing increases the surface area of the plant for the availability of all secondary metabolites to be contacted with the diseases causing germs (No, 2014).

The present study documented various ways of administration and applications of herbal treatments used by traditional healers of the study area. In the study area, plant powder was mainly taken with water and rarely with traditional beverages, coffee, milk, and tea orally. This could be due to the high prevalence of internal disorders. This finding is similar to results reported by various researchers in Ethiopia and elsewhere (Lulekal et al., 2013; Birhanu, Endale, et al., 2015; Aziz et al., 2017).

\subsection{Fidelity level values, preference, and direct matrix ranking exercises}

The calculation of fidelity level of medicinal plants revealed that Ocimum lamiifolium, Albizia anthelmintica, Phytolacca dodecandra, Dichrostachys cinerea, Andrachne aspera, and Psydrax schimperiana had high fidelity levels. According to Trotter and Logan (1986), fidelity level is a measure of the healing potential of medicinal plants. Thus, medicinal plants having a high-fidelity level indicates that they are supposed to be effective in their curing ability.

The preference ranking exercise helped in determining which medicinal plant species are most used to treat cellulitis (contact dermatitis). As the result, Dichrostachys cinerea (L.) Wight \& Arn., Clematis simensis Fresen, Premna schimperii Engl. Zehneria scabra Sond. and Rhus natalensis Bernh. had the highest scores and were identified as the most effective treatments for this disease. Future research on the bioactive components of these species against cellulitis can lead to good outcomes.

Due to factors related to 'modernization,' the younger generation of Ensaro district showed a lack of interest in traditional treatments. Similar findings have been observed for other Ethiopian cultural groups (Lulekal et al., 2013). It was also shown that traditional healers maintain the highest level of secrecy when dealing with medicinal plant information. Furthermore, they make every effort to keep the information within the family circle secret. These facts, combined with the lack of any written documentation on medicinal plants in the study area, indicated a threat to the future use of medicinal plants.

The outcome of the direct matrix ranking exercise revealed the highest-ranking values for Cordia Africana, Ziziphus spina-christi, and Olea europaea subsp. Cuspidata for their non-medicinal purposes. Overexploitation for timber production, farm implements, construction, and fuelwood are the main factors for the threatening of these medicinal plant species in the study area. The result indicated, therefore, urgent remedial measures should be required to save the rapidly eroding medicinal plants of the study area. in Ankober district of Ethiopia, Lulekal et al. (2013) found a similar pattern of maximum utilization of medicinal plants for other non-medicinal purposes.

\subsection{Comparative review results from similar studies}

In this study, all known medicinal plant species were searched in published research articles from Ethiopia (Gedif \& Hahn, 2003; Giday et al., 2003; Giday et al., 2007; Teklehaymanot et al., 2007; Lulekal et al., 2008; Giday et al., 2009; Mesfin et al., 2009; Lulekal et al., 2013; Chekole, 2017; Tefera \& Yihune, 2019), Kenya (Jeruto et al., 2008; Okello et al., 2010; Muthee et al., 2011; Nyang'au et al., 2017; Orech \& Schwarz, 2017; Lako et al., 2020; Omara, 2020), Burkina Faso (Nadembega et al., 2011) and South Sudan (Lako et al., 2020). From this review, 133 recorded medicinal plant species in this study are likely also to be found in other parts of Ethiopia and other countries with similar uses, parts use, conditions of preparation, diseases treated. Sixty-one of them have similar functions and 72 have different functions. The rest 9 reported medicinal plant species (Abutilon longicuspe Hochst. ex A.Rich, Adenia cissampeloides (Planch. ex Hook.) Harms, Duranta erecta L., Emilia sonchifolia (L.) DC. ex DC., Psydrax schimperiana (A.Rich.) Bridson, Snowdenia polystachya (Fresen.) Pilg., Solanum dulcamara L., Symphytum officinale L, and Veronica anagallis-aquatica L.) Wedd.) are reported only by this study as far as our literature search.

\section{Conclusions}

This is the first ethnobotanical report on medicinal uses of plants by the communities of Ensaro district, North Shewa Zone, Amhara Regional State, Ethiopia. According to the findings of this study, the residents of Ensaro district have deep knowledge about which plants to collect for the treatment of common human ailments and diseases, as well as where they grow. The findings of this study supported up the idea that indigenous knowledge of using medicinal plants is still alive in highly degraded areas. This finding is also revealed that traditional healers in Ensaro district collected medicinal plants from wild habitats. Several researchers have documented many of these plants, but a few are new for Ethiopia and other countries. This study provides baseline data to warrant further studies on the actual plants being used, and has prioritized plants for further biological and toxicological screening as well as promoting their conservation and sustainable use. To make sustainable use of these medicinal plants in the study area, local people must collaborate with natural resource administrators to manage them before they become seriously threatened. Both ex-situ and In-situ conservation strategies should be combined and employed urgently for optimal conservation results. 


\section{Declarations}

\section{Ethics approval and consent participant}

Permission to conduct the ethnobotanical study was obtained from the district administration and village administrators in the study area. The purpose of the study was explained to all participants, and they agreed to provide information

\section{Funding}

The ministry of higher education, Ethiopian public health institute and Debere Berhan University supported this study.

\section{Availability of data and materials}

The authors declare that all other data supporting the findings of this study are available within the article and its supplementary information files.

\section{Competing interest}

The authors have not declared any conflicts of interest.

\section{Authors' contributions}

Asaye Asfaw conceived the idea of doing this research, conducted the interviews, collected samples, outlined and wrote the manuscript. Ermias Lulekal was involved in the identification of collected plant materials and searching for the fund. Asfaw Debella, Aby Abebe and Sileshi Degu participated in the planning and preparation of semi-structured questions. Tamrat Bekele was revising it critically for important intellectual content. All authors have made substantial contributions to the analysis, interpretation of data and writing of the manuscript. All authors read and approved the final manuscript.

\section{Acknowledgement}

The authors appreciate and express their thanking words to officials of Ensaro woreda who facilitated the study and the community members that participated during the interviews. Without their contribution, this study would have been impossible. The first author thanks the scholarship from Debre Berhan University. EPHI (Ethiopian people health institute, period 2019/2021) who gave the financial support is acknowledged.

\section{References}

1. Abebe BA, Chane Teferi S. (2021). Ethnobotanical Study of Medicinal Plants Used to Treat Human and Livestock Ailments in Hulet Eju Enese Woreda, East Gojjam Zone of Amhara Region, Ethiopia. Evidence-Based Complementary and Alternative Medicine, 2021.

2. Abebe D. Traditional Medicine in Ethiopia: The Attempts Being Made to Promote It for Effective and Better Utilization. SINET. 1986;9(Suppl.):61-9.

3. Abebe D, Ayehu A. (1993). Medicinal Plants and Enigmatic Health Practices of Northern Ethiopia.

4. Abebe D, Zewdu M, Demissei A. (2001). Conservation and Sustainable Use of Medicinal Plants in Ethiopia, Proceedings of the National Workshop, 28 April-01 May 1998.

5. Adam M, Ahmed AA, Yagi A, Yagi S. (2020). Ethnobotanical Investigation on Medicinal Plants Used against Human Ailments in Erkowit and Sinkat Areas, Eastern Sudan. Biodiversitas Journal of Biological Diversity, 21(7).

6. Agisho H, Osie M, Lambore T. Traditional Medicinal Plants Utilization, Management and Threats in Hadiya Zone, Ethiopia. J Med Plant. 2014;2(2):94108.

7. Alemayehu G, Asfaw Z, Kelbessa E. Plant Diversity and Ethnobotany in Berehet District, North Shewa Zone of Amhara Region (Ethiopia) with Emphasis on Wild Edible Plants. Journal of medicinal plants studies. 2015;3(6):93-105.

8. Alexiades MN, Sheldon JW. (1996). Selected Guidelines for Ethnobotanical Research: A Field Manual.

9. Asefa M, Cao M, He Y, Mekonnen E, Song X, Yang J. Ethiopian Vegetation Types, Climate and Topography. Plant Diversity. 2020;42(4):302-11.

10. Assefa B, Glatzel G, Buchmann C. Ethnomedicinal Uses of Hagenia Abyssinica (Bruce) Jf Gmel. Among Rural Communities of Ethiopia. J Ethnobiol Ethnomed. 2010;6(1):1-10.

11. Assen Y, Woldearegay M, Haile A. (2021). An Ethnobotanical Study of Medicinal Plants in Kelala District, South Wollo Zone of Amhara Region, Northeastern Ethiopia. Evidence-Based Complementary and Alternative Medicine, 2021.

12. Awas T. Endemic Plants of Ethiopia: Preliminary Working List to Contribute to National Plant Conservation Target. Addis Ababa: Ethiopian Institute of Biodiversity, 2009

13. Aziz MA, Khan AH, Adnan M, Izatullah I. Traditional Uses of Medicinal Plants Reported by the Indigenous Communities and Local Herbal Practitioners of Bajaur Agency, Federally Administrated Tribal Areas, Pakistan. J Ethnopharmacol. 2017;198:268-81.

14. Bekele E. (2007). Study on Actual Situation of Medicinal Plants in Ethiopia. Addis Ababa: Prepared for Japan Association for International Collaboration of Agriculture and Forestry.

15. Birhanu T, Abera D, Ejeta E, Nekemte E. Ethnobotanical Study of Medicinal Plants in Selected Horro Gudurru Woredas, Western Ethiopia. Journal of Biology Agriculture Healthcare. 2015;5(1):83-93.

16. Birhanu Z, Endale A, Shewamene Z. An Ethnomedicinal Investigation of Plants Used by Traditional Healers of Gondar Town, North-Western Ethiopia. Journal of medicinal plants studies. 2015;3(2):36-43. 
17. Chekole G. Ethnobotanical Study of Medicinal Plants Used against Human Ailments in Gubalafto District, Northern Ethiopia. J Ethnobiol Ethnomed. 2017;13(1):1-29.

18. Chekole G, Asfaw Z, Kelbessa E. Ethnobotanical Study of Medicinal Plants in the Environs of Tara-Gedam and Amba Remnant Forests of Libo Kemkem District, Northwest Ethiopia. J Ethnobiol Ethnomed. 2015;11(1):1-38.

19. Cherinet A, Mekonnen Z. Comparing Farmers' Perception of Climate Change and Variability with Historical Climate Data: The Case of Ensaro District, Ethiopia. International Journal of Environmental Sciences Natural Resources. 2019;17(4):114-20.

20. Chowdhury AHK, Shahriar MH, Rahman MS, Uddin MP, Al-Amin M, Rahman MM,... Azad A. Home Remedies of Rural Folks: A Study in Kadipur Village of Chuadanga District, Bangladesh. World Journal of Pharmacy Pharmaceutical Sciences (WJPPS). 2015;4(1):171-82.

21. Cotton C. (1996). Ethnobotany: Principles and Applications (047195537X). Retrieved from.

22. d'Avigdor E, Wohlmuth H, Asfaw Z, Awas T. The Current Status of Knowledge of Herbal Medicine and Medicinal Plants in Fiche, Ethiopia. J Ethnobiol Ethnomed. 2014;10(1):1-33.

23. Deffar G. (1998). Non-Wood Forest Products in Ethiopia.

24. Ernst E. Prevalence of Use of Complementary/Alternative Medicine: A Systematic Review. Bull World Health Organ. 2000;78:258-66.

25. Fenetahun Y, Eshetu G, Worku A, Abdella T. A Survey on Medicinal Plants Used by Traditional Healers in Harari Regional State, East Ethiopia. Journal of medicinal plants studies. 2017;5(1):85-90.

26. Friedman J, Yaniv Z, Dafni A, Palewitch D. A Preliminary Classification of the Healing Potential of Medicinal Plants, Based on a Rational Analysis of an Ethnopharmacological Field Survey among Bedouins in the Negev Desert, Israel. J Ethnopharmacol. 1986;16(2-3):275-87.

27. Gazzaneo LRS, De Lucena RFP, de Albuquerque UP. Knowledge and Use of Medicinal Plants by Local Specialists in an Region of Atlantic Forest in the State of Pernambuco (Northeastern Brazil). J Ethnobiol Ethnomed. 2005;1(1):1-8.

28. Gebrelibanos M, Asres K, Veeresham C. In Vitro Radical Scavenging Activity of the Leaf and Bark Extracts of Senna Singueana (Del). Lock. Ethiopian Pharmaceutical Journal. 2008;25:77-84.

29. Gedif T, Hahn H-J. The Use of Medicinal Plants in Self-Care in Rural Central Ethiopia. J Ethnopharmacol. 2003;87(2-3):155-61.

30. Getaneh S, Girma Z. An Ethnobotanical Study of Medicinal Plants in Debre Libanos Wereda, Central Ethiopia. African Journal of Plant Science. 2014;8(7):366-79.

31. Giday M, Asfaw Z, Elmqvist T, Woldu Z. An Ethnobotanical Study of Medicinal Plants Used by the Zay People in Ethiopia. J Ethnopharmacol. 2003;85(1):43-52.

32. Giday M, Asfaw Z, Woldu Z. Medicinal Plants of the Meinit Ethnic Group of Ethiopia: An Ethnobotanical Study. J Ethnopharmacol. 2009;124(3):513-21.

33. Giday M, Teklehaymanot T, Animut A, Mekonnen Y. Medicinal Plants of the Shinasha, Agew-Awi and Amhara Peoples in Northwest Ethiopia. J Ethnopharmacol. 2007;110(3):516-25.

34. Hailemariam T, Demissew S, Asfaw Z. An Ethnobotanical Study of Medicinal Plants Used by Local People in the Lowlands of Konta Special Woreda, Southern Nations, Nationalities and Peoples Regional State, Ethiopia. J Ethnobiol Ethnomed. 2009;5(26):4269-4265.

35. Hamilton A. (2003). Medicinal Plants and Conservation: Issues and Approaches. International Plants Conservation Unit, WWF-UK, 51.

36. Hartmann I. Cultural Diversity in Ethiopia and Its Impact on Local Economies and Biodiversity. In: The Future of Drylands. Springer; 2008. pp. 145-60.

37. Heinrich M. Ethnobotany and Its Role in Drug Development. Phytotherapy Research: An International Journal Devoted to Pharmacological Toxicological Evaluation of Natural Product Derivatives. 2000;14(7):479-88.

38. Hosseini SH, Bibak H, Ghara AR, Sahebkar A, Shakeri A. Ethnobotany of the Medicinal Plants Used by the Ethnic Communities of Kerman Province, Southeast Iran. J Ethnobiol Ethnomed. 2021;17(1):1-35.

39. Jeruto P, Lukhoba C, Ouma G, Otieno D, Mutai C. An Ethnobotanical Study of Medicinal Plants Used by the Nandi People in Kenya. J Ethnopharmacol. 2008;116(2):370-6.

40. Kebede E, Mengistu M, Serda B. Ethnobotanical Knowledge of Pastoral Community for Treating Livestock Diseases in Somali Regional State, Eastern Ethiopia. Trop Anim Health Prod. 2018;50(6):1379-86.

41. Kelbessa E, Demissew S. Diversity of Vascular Plant Taxa of the Flora of Ethiopia and Eritrea. Ethiopian Journal of Biological Sciences. 2014;13(Supp.):37-45.

42. Kelemu T, Wolde W. Ethnobotanical Study of Indigenous Knowledge on Medicinal Plants Used to Treat Diseases in Selected Districts of Amhara Regional State, Ethiopia. Journal of Medicinal Plants Research. 2018;12(29):528-36.

43. Kidane L, Gebremedhin G, Beyene T. Ethnobotanical Study of Medicinal Plants in Ganta Afeshum District, Eastern Zone of Tigray, Northern Ethiopia. J Ethnobiol Ethnomed. 2018;14(1):1-19.

44. Kim J, De Jesus O. (2021). Medication Routes of Administration.

45. Kiringe JW. A Survey of Traditional Health Remedies Used by the Maasai of Southern Kaijiado District, Kenya. Ethnobotany Research Applications. 2006;4:061-74.

46. Lako JDW, Sube KLL, Lumori CSG, Yengkopiong JP, Utong JAM, Binyason SA,.. . Kheiralla AH. Diversity and Distribution of Medicinal Plants in the Republic of South Sudan. World Journal of Advanced Research Reviews. 2020;7(1):018-31.

47. Lulekal E, Asfaw Z, Kelbessa E, Van Damme P. Ethnomedicinal Study of Plants Used for Human Ailments in Ankober District, North Shewa Zone, Amhara Region, Ethiopia. J Ethnobiol Ethnomed. 2013;9(1):1-13. 
48. Lulekal E, Asfaw Z, Kelbessa E, Van Damme P. Ethnoveterinary Plants of Ankober District, North Shewa Zone, Amhara Region, Ethiopia. J Ethnobiol Ethnomed. 2014;10(1):1-19.

49. Lulekal E, Kelbessa E, Bekele T, Yineger H. An Ethnobotanical Study of Medicinal Plants in Mana Angetu District, Southeastern Ethiopia. J Ethnobiol Ethnomed. 2008;4(1):1-10.

50. Martin G. Ethnobotany-a Manual of Methods. London: Chapman et Hall; 1995.

51. Martin G. (1995). Ethnobotany: A Methods Manual, Chapman Y Hall. Nowy Jork.

52. Mebrat W, Gashaw T. Threats of Woody Plant Species Diversity and Their Conservation Techniques in Ethiopia. European Journal of Botany Plant Science Phytology. 2013;3:10-7.

53. Mesfin F, Demissew S, Teklehaymanot T. An Ethnobotanical Study of Medicinal Plants in Wonago Woreda, Snnpr, Ethiopia. J Ethnobiol Ethnomed. 2009;5(1):1-18.

54. Moges A, Moges Y. (2019). Ethiopian Common Medicinal Plants: Their Parts and Uses in Traditional Medicine-Ecology and Quality Control. Plant ScienceStructure, Anatomy and Physiology in Plants Cultured in Vivo and in Vitro.

55. Muthee J, Gakuya D, Mbaria J, Kareru P, Mulei CM, Njonge F. Ethnobotanical Study of Anthelmintic and Other Medicinal Plants Traditionally Used in Loitoktok District of Kenya. J Ethnopharmacol. 2011;135(1):15-21.

56. Nadembega P, Boussim JI, Nikiema JB, Poli F, Antognoni F. Medicinal Plants in Baskoure, Kourittenga Province, Burkina Faso: An Ethnobotanical Study. J Ethnopharmacol. 2011;133(2):378-95.

57. No JG. Changes in Secondary Metabolite Contents Following Crude Drug Preparation. Procedia Chem. 2014;13:57-62.

58. Nyang'au HO, Maingi J, Kebira A. The Efficacy of Some Medicinal Plants Used Locally within Transmara West. Narok County, Kenya against Selected Enterobacteria and Candida; 2017.

59. Okello S, Nyunja R, Netondo GW, Onyango JC. (2010). Ethnobotanical Study of Medicinal Plants Used by Sabaots of Mt. Elgon Kenya. African journal of traditional, complementary and alternative medicines, 7(1).

60. Omara T. (2020). Antimalarial Plants Used across Kenyan Communities. Evidence-Based Complementary and Alternative Medicine, 2020.

61. Orech F, Schwarz J. Ethno-Phytotherapeutic Remedies Used in Meat, Milk, and Blood Products by the Maasai People of Kenya. South African Journal of Botany. 2017;108:278-80.

62. Pascaline J, Charles M, George O, Lukhoba C. An Inventory of Medicinal Plants That the People of Nandi Use to Treat Malaria. J Anim Plant Sci. 2011;9:1192-200.

63. Prashanth Kumar G, Shiddamallayya N. Survey of Wild Medicinal Plants of Hassan District, Karnataka. J Med Plants Stud. 2016;4(1):91-102.

64. Tardío J, Pardo-de-Santayana M. Cultural Importance Indices: A Comparative Analysis Based on the Useful Wild Plants of Southern Cantabria (Northern Spain). Econ Bot. 2008;62(1):24-39.

65. Tefera T, Yihune M. Ethnobotanical Study on Medicinal Plants Used by Indigenous People in Tenta District, South Wollo, Ethiopia. Journal of Medicinal Plants Research. 2019;13(2):47-54.

66. Teklay A, Abera B, Giday M. An Ethnobotanical Study of Medicinal Plants Used in Kilte Awulaelo District, Tigray Region of Ethiopia. J Ethnobiol Ethnomed. 2013;9(1):1-23.

67. Teklehaymanot T, Giday M. Ethnobotanical Study of Medicinal Plants Used by People in Zegie Peninsula, Northwestern Ethiopia. Journal of ethnobiology Ethnomedicine. 2007;3(1):1-11.

68. Teklehaymanot T, Giday M, Medhin G, Mekonnen Y. Knowledge and Use of Medicinal Plants by People around Debre Libanos Monastery in Ethiopia. J Ethnopharmacol. 2007;111(2):271-83.

69. Tesfaye S, Belete A, Engidawork E, Gedif T, Asres K. (2020). Ethnobotanical Study of Medicinal Plants Used by Traditional Healers to Treat Cancer-Like Symptoms in Eleven Districts, Ethiopia. Evidence-Based Complementary and Alternative Medicine, 2020.

70. Trotter RT, Logan MH. (1986). Informant Consensus: A New Approach for Identifying Potenially Effective Medicinal Plants.

71. Trotter RT, Logan MH. Informant Consensus: A New Approach for Identifying Potentially Effective Medicinal Plants. In: Plants in Indigenous Medicine \& Diet. Routledge; 2019. pp. 91-112.

72. Tuasha N, Petros B, Asfaw Z. Medicinal Plants Used by Traditional Healers to Treat Malignancies and Other Human Ailments in Dalle District, Sidama Zone, Ethiopia. J Ethnobiol Ethnomed. 2018;14(1):1-21.

73. Vivero JL, Kelbessa E, Demissew S. The Red List of Endemic Trees \& Shrubs of Ethiopia and Eritrea. Fauna \& Flora International; 2005.

74. Yang Y, Luo X, Wei W, Fan Z, Huang T, Pan X. Analysis of Leaf Morphology, Secondary Metabolites and Proteins Related to the Resistance to Tetranychus Cinnabarinus in Cassava (Manihot Esculenta Crantz). Sci Rep. 2020;10(1):1-13.

75. Yineger H, Kelbessa E, Bekele T, Lulekal E. Ethnoveterinary Medicinal Plants at Bale Mountains National Park, Ethiopia. J Ethnopharmacol. 2007;112(1):55-70.

76. Yirga G, Teferi M, Gidey G, Zerabruk S. An Ethnoveterinary Survey of Medicinal Plants Used to Treat Livestock Diseases in Seharti-Samre District, Northern Ethiopia. African Journal of Plant Science. 2012;6(3):113-9.

77. Yohannis SW, Zemede A, Ensermu K. Ethnobotanical Study of Medicinal Plants Used by Local People in Menz Gera Midir District, North Shewa Zone, Amhara Regional State, Ethiopia. Journal of Medicinal Plants Research. 2018;12(21):296-314.

78. Zerabruk S, Yirga G. Traditional Knowledge of Medicinal Plants in Gindeberet District, Western Ethiopia. South African Journal of Botany. 2012;78:165-9. 
Figures
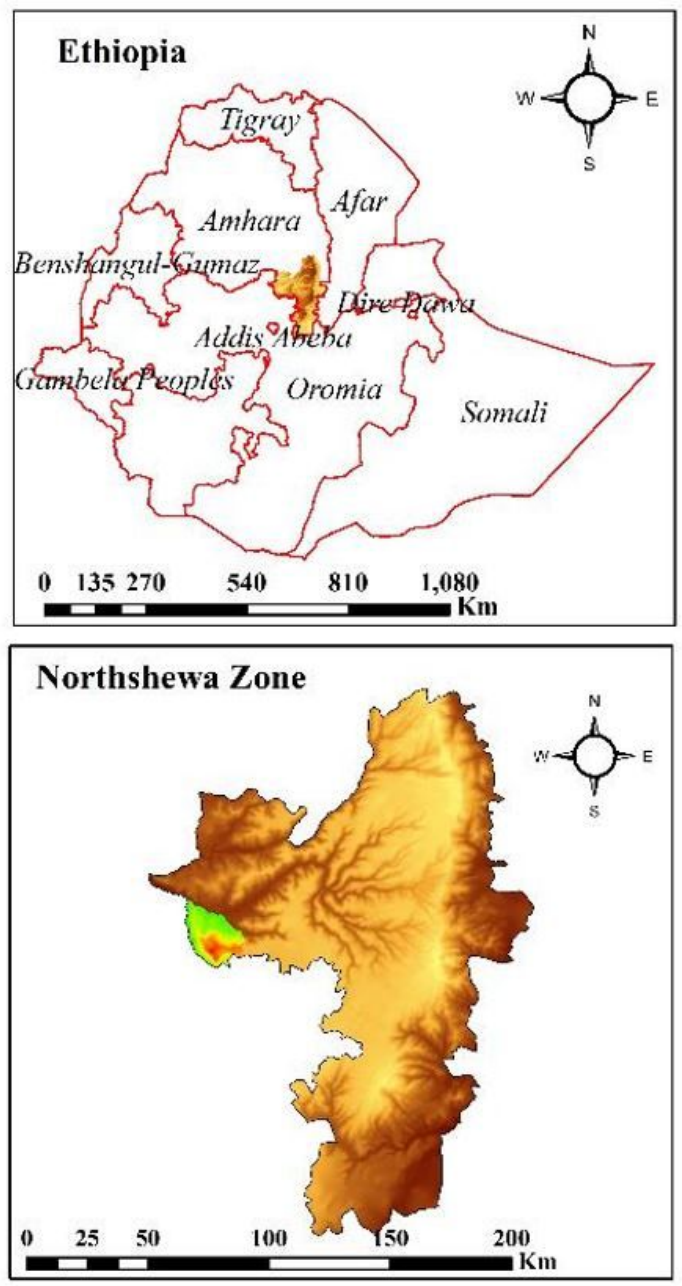

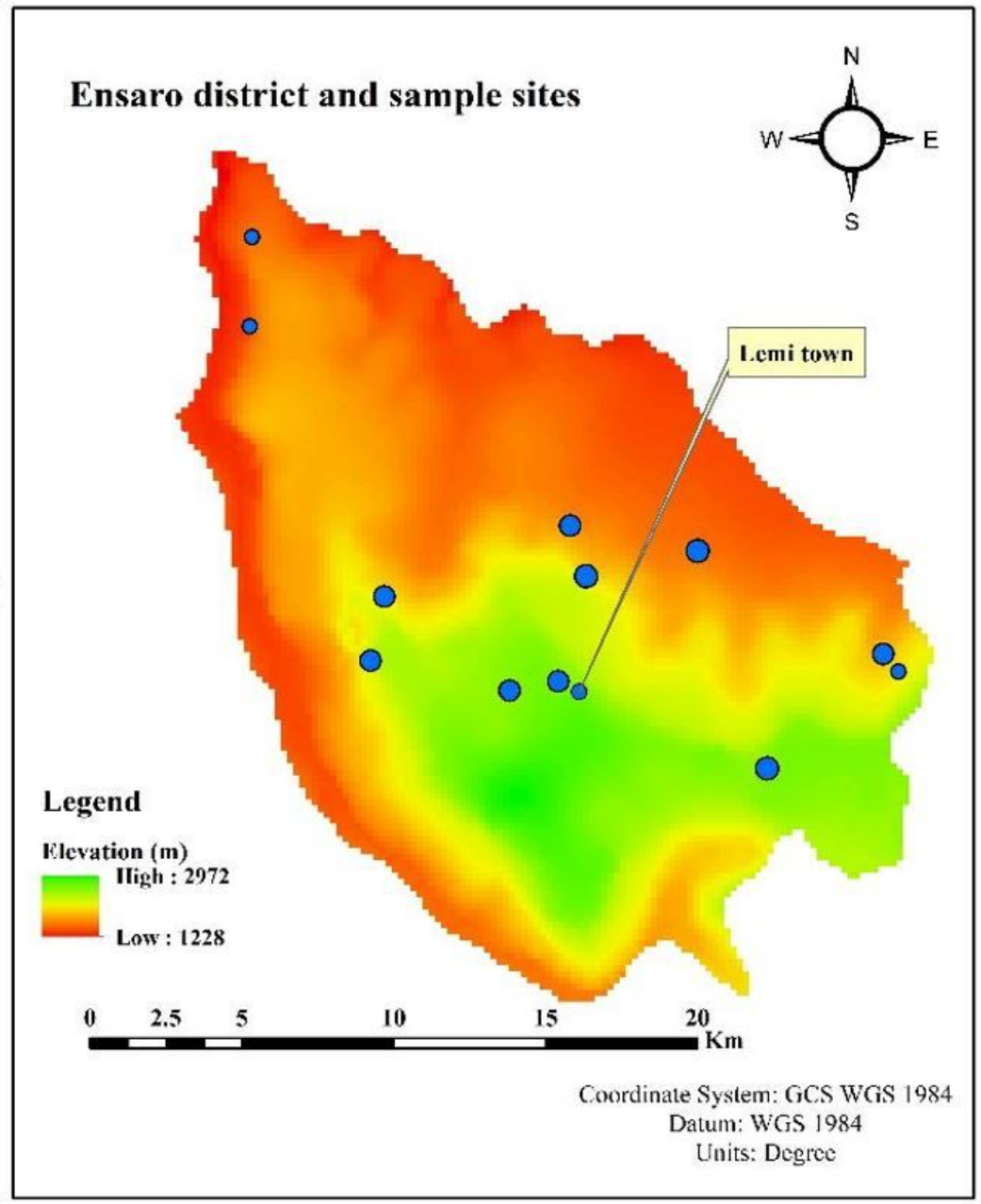

Figure 1

A Map of Ethiopia showing Amhara Region and the study district 


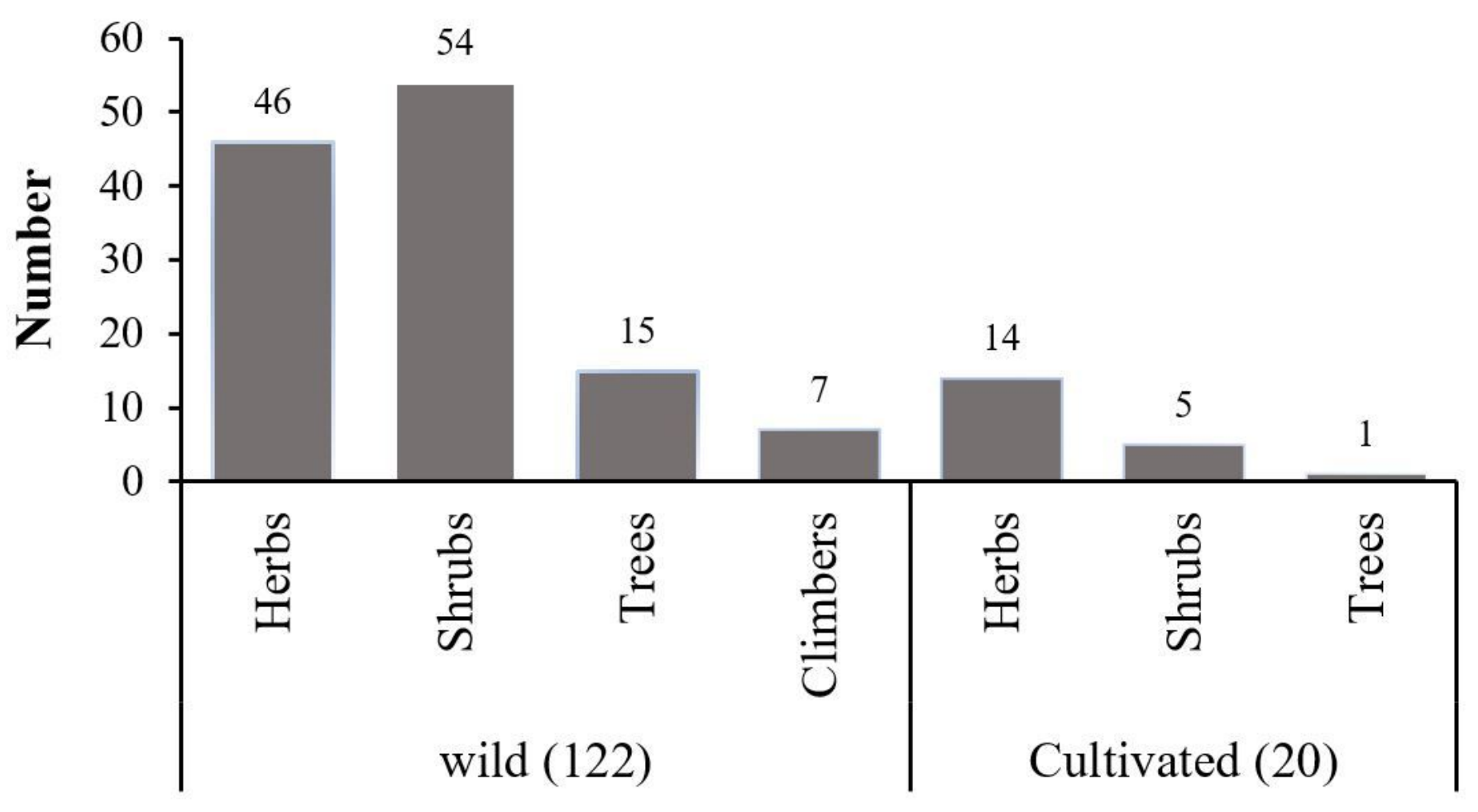

Life forms of wild and cultivated medicinal plants

Figure 2

The life forms of wild and cultivated medicinal plants in the study area.

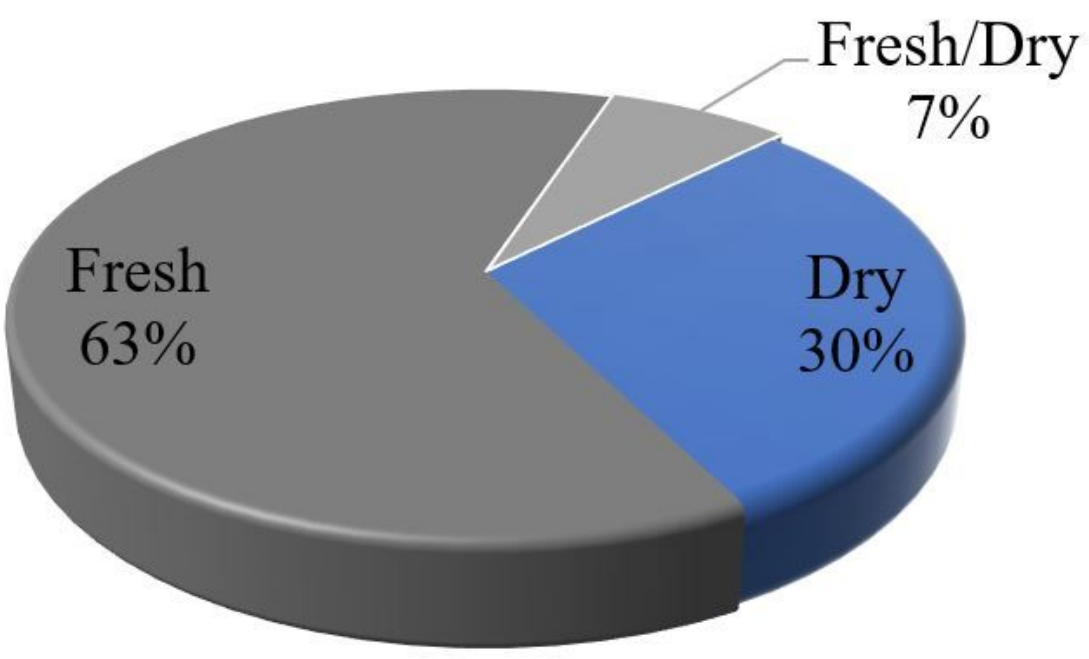

Figure 3

Conditions of traditional herbal medicines preparation 


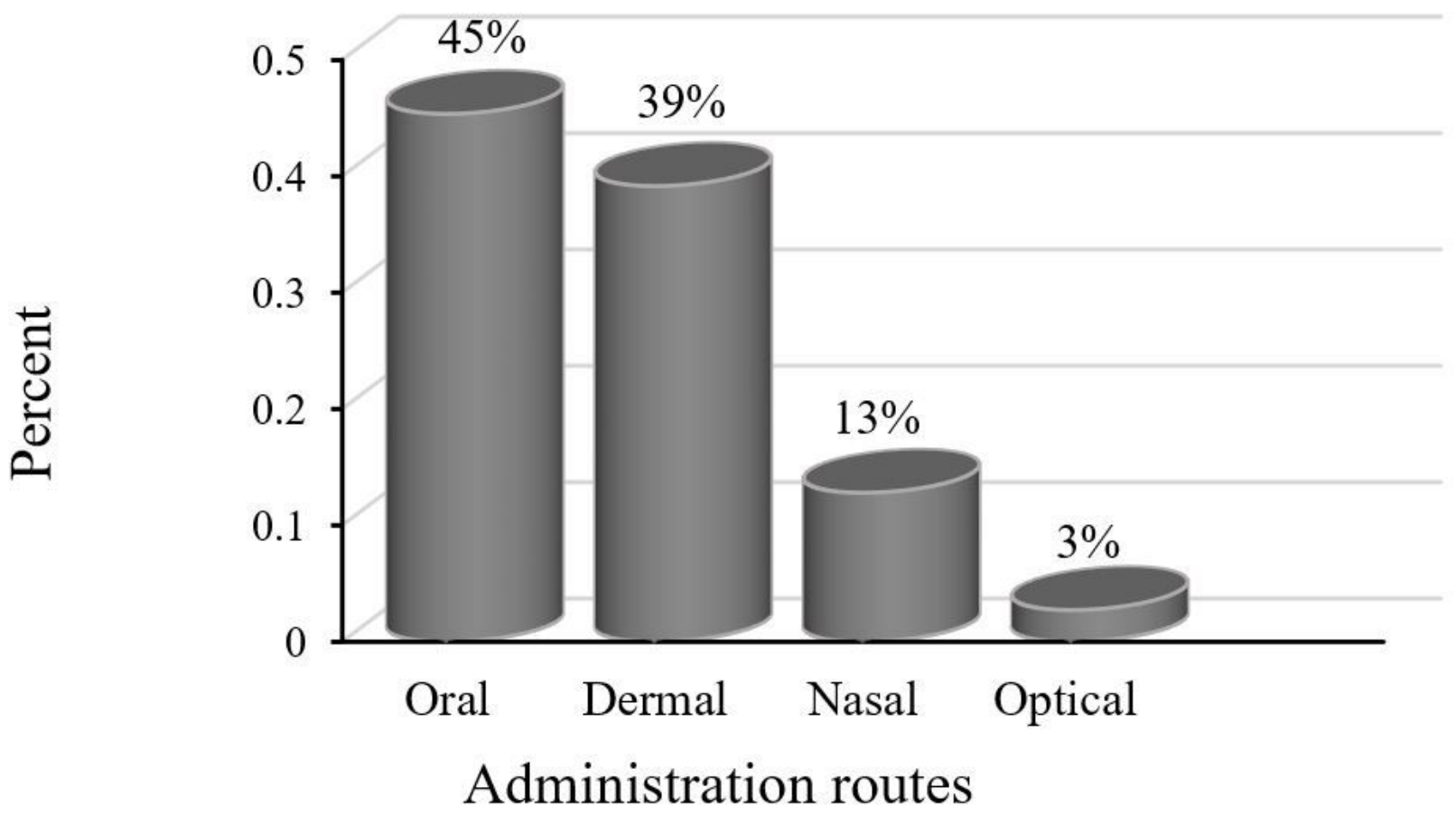

Figure 4

Showing the frequency percentage of administration route 